\title{
Resource Allocations for Symbiotic Radio with Finite Block Length Backscatter Link
}

\author{
Zheng Chu, Wanming Hao, Pei Xiao, Mohsen Khalily, and Rahim Tafazolli
}

\begin{abstract}
This paper exploits a generic downlink symbiotic radio (SR) system, where a Base Station (BS) establishes a direct (primary) link with a receiver having an integrated backscatter device (BD). In order to accurately measure the backscatter link, the backscattered signal packets are designed to have finite blocklength. As such, the backscatter link in this SR system employs the finite block-length channel codes. According to different types of the backscatter symbol period and transmission rate, we investigate the non-cooperative and cooperative SR (i.e., NSR and CSR) systems, and derive their average achievable rate of the direct and backscatter links, respectively. We formulate two optimization problems, i.e., transmit power minimization and energy efficiency maximization. Due to the non-convex property of these formulated optimization problems, the semidefinite programming (SDP) relaxation and the successive convex approximation (SCA) are considered to design the transmit beamforming vector. Moreover, a low-complexity transmit beamforming structure is constructed to reduce the computational complexity of the SDP relaxed solution. Finally, the simulation results are demonstrated to validate the proposed schemes.
\end{abstract}

Index Terms-Symbiotic radio (SR), backscatter, finite blocklength channel codes, semi-definite programming (SDP), successive convex approximation (SCA)

\section{INTRODUCTION}

As one of the emerging technologies in the future wireless network, internet of things (IoT), significantly improves massive connectivity for a large number of IoT devices. However, with an explosively increasing number of these devices, the wireless network will suffer from energy-constrained and limited radio spectrum issues. It leads to the fact that the devices in an IoT network cannot always connect with the IoT server over long time period and the fact that for most IoT applications, such as those enabling the smart city, the device life cycle is 10 to 20 years or more, which poses extremely demanding battery life constraints. Thus, energy efficiency and radio spectrum resource has been main challenges in IoT networks [1], [2].

Ambient backscatter communication (AmBC) were proposed to fix the energy-constrained issue in IoT networks, where a passive backscatter device (BD) carries on ambient

This work was supported by the U.K. Engineering and Physical Sciences Research Council under Grant EP/P008402/2 and EP/P03456X/1. The authors also would like to acknowledge the support of the University of Surrey 5GIC (http://www.surrey.ac.uk/5gic) members for this work. The work of W. Hao was supported by China Post-Doctoral Science Foundation under Grant 2019M662528.

Z. Chu, P. Xiao, M. Khalily, and R. Tafazolli are working with the Institute for Communication Systems, University of Surrey, Guildford GU2 7XH, United Kingdom. (Email: andrew.chuzheng7@gmail.com; p.xiao@surrey.ac.uk; m.khalily@surrey.ac.uk; r.tafazolli@surrey.ac.uk)

W. Hao is working with the School of Information Engineering, Zhengzhou University, Zhengzhou, 450001, China. (Email: wmhao@hotmail.com) radio frequency (RF) signals (i.e., cellular, $\mathrm{WiFi}$, and $\mathrm{TV}$ signals) via modulating its own information [3]. This technique is different from the conventional IoT devices, which utilize active RF components, i.e., converters and oscillators, etc., leading to high cost and energy consumption [2]. The passive BD yields a great reduction of the energy consumption at the IoT network since no active RF components are required at the IoT device [4]. However, the backscatter link in AmBC is vulnerable to the interference from the direct link due to inefficient utilization in spectrum such that the backscatter transmission may suffer from a performance degradation [5]. In order to circumvent this problem, symbiotic radio (SR) can be considered as a promising solution, which is based on a cooperative AmBC system and aims to integrate the BD with a direct transmission [2], [6]. Unlike the aforementioned traditional AmBC systems, the direct link is also known as the primary link, which shares the radio spectrum with the backscatter link such that the receiver simultaneously decodes the messages from the transmitter and the BD. Radio frequency identification (RFID), one typical backscatter communication system is a good application in a IoT network [2], [7]. The BDs in the IoT setup evolves the active Bluetooth or LoRa devices to collect the information transmitted from the BDs by aligning the frequency of the original signal emitted by the transmitter [8], [9].

\section{A. State-of-the-Art}

Some classical techniques are employed to investigate the AmBC systems in the existing works [10]-[14]. In [10], novel error-floor-free detectors were investigated to deal with the interference of the direct link at the multi-antenna reader. Also, a joint design for the backscatter waveform and receiver detector was exploited to investigate the AmBC system by capitalizing the ambient orthogonal frequency division multiplexing (OFDMA) modulated signal [11]. Cooperative AmBC system was proposed in [12], where the multi-antenna cooperative receiver is developed to recover the information from the RF source as well as the BD in different fading channels. In [13], a novel spectrum sharing scheme was exploited in the AmBC system to maximize the BD's ergodic capacity, which is to jointly design the RF source's power allocation and the BD's reflection coefficient. Moreover, a novel backscatter multiplicative multiple-access channel (BMMAC) was proposed [14]. Particularly, the achievable rate region of the BM-MAC has been analyzed to show that it is strictly larger than that of the conventional time-division multiple-access (TDMA) scheme when the channel state information (CSI) in the direct link is much better than that of 
the backscatter link. Hence, the multiplicative multiple-access scheme can improve the throughput for AmBC systems [14].

Recently, the SR system has attracted increasing attention [15]-[20]. In [15], a resource allocation scheme in the SR system was investigated in the fading channel, where the power allocation at the primary transmitter (PT) and the reflection coefficient at the BD are jointly designed to maximize the ergodic weighted sum rate of the direct and backscatter links in the long-term and short-term transmit power budget over the fading states, respectively. The authors in [16] investigated resource allocation scheme in a novel SR paradigm, i.e., cooperative AmBC, where three practical SR schemes (i.e., commensal, parasitic, and competitive) have been exploited, respectively, to derive the achievable rates for both direct and backscatter links for the fading state. Additionally, the power allocation was optimally achieved for each SR scheme under the average power budget. Integration a SR system with a passive full duplex (FD) BD was investigated in [17], [18], where the FD BD is considered as a parasitic transmission of the direct link. The multi-antenna PT connects to the primary receiver $(\mathrm{PR})$ and the $\mathrm{BD}$, integrating with the passive transmission from the BD to the PR. The FD BD can extract a portion of the original signal from the PT to decode the information, and simultaneously transmits its own information to the receiver via backscattering the remaining portion of the original signal [17], [18]. Integration of non-orthogonal multiple access (NOMA) into the SR system was presented to form a symbiotic network (i.e., including cellular and IoT networks) [19]. Specifically, a Base Station (BS) broadcasts the NOMA signal to two cellular users, while a BD modulates its own information over the original signals from the BS to a cellular user via the passive radio frequency. Very recently, the deep reinforcement learning (DRL) techniques have been introduced into the SR system [20], which can obtain the full real-time channel information. Particularly, the current information can be predicted using the previous information so as to make an informed decision.

Although the above works have investigated the SR system, which was integrated with some promising techniques, these are based on the premise of the conventional Shannon's capacity, i.e., ergodic capacity or outage capacity metric, which is typically considered for the long transmit packet lengths. However, the BD is generally a low-power IoT device, which utilizes limited power to modulate its own signal to reflect the intended signal to the receiver. This may increase the payload of the BD with Shannon's capacity metric. From the information-theoretical perspective, the Shannon's capacity does not help us gain an accurate insight into the engineering system design with finite code block-length [21]. Thus, the achievable rate in finite block-length regime was introduced to provide a new performance metric for finite packet-length transmission, which is measured by incurring a penalty (i.e., channel dispersion) on ergodic capacity [21]. In recent years, finite packet-length transmission has been investigated in several typical scenarios, which mainly focus on the metrics of ultra-reliable and low-latency features. In [22], new wireless protocol designs have been recently developed for finite packet-length transmissions in ultra-reliable and low-latency communication (URLLC) networks. Additionally, short packet transmission were studied in the relay-enabled URLLC network in [23], [24], where accurate performance analysis and optimization were discussed. Those works manifested the advantage of applying relaying in 5G URLLC networks over the direct transmission case (without relaying). Recently, a novel multiple access technique, namely, NOMA, was introduced and analyzed to measure ultra-reliable and low-latency features, by applying the coding rate in the finite block-length regime to enhance the system fairness and spectral efficiency [25]. Against this background, it is an interesting idea to incorporate the finite block-length channel codes into the SR system. Specifically, the achievable rate in the backscatter link is measured to be in the finite block-length regime. To the best of our knowledge, there is no existing work to focus on the resource allocation with the finite block-length backscatter link, which motivates the work in this paper.

In this paper, we consider a generic downlink multipleinput single-output (MISO) SR system, where a BS has a communication link with a receiver, and the BD modulates its signal to reflect the intended signal from the BS to the receiver. We summarize the contributions of this paper as follows:

1) We first investigate two SR system models: noncooperative $S R$ (NSR) and cooperative $S R$ (CSR) according to different types of the backscatter symbol period and transmission rate. In addition, the backscatter link in the NSR system introduces the interference to the direct link, while the receiver in the CSR system can decode the information of the direct link with a multipath signal of the backscatter link, resulting in both direct and backscatter links being beneficial to each other.

2) Next, the achievable rate of the direct link is characterized by the coexistence with the interference of the backscatter link in the NSR system and with a multipath component of the backscatter link in the CSR system. We also investigate the achievable rate of the backscatter link in the finite block-length regime to gain a practical engineering insight in the case when the receiver employs the successive interference cancellation (SIC) to remove the direct link signal.

3) We formulate two optimization problems, transmit power minimization and energy efficiency maximization to exploit these two SR systems. Due to the nonconvexity of the transmit power minimization problem, we first adopt a semi-definite programming (SDP) relaxation and a successive convex approximation (SCA) approach to make it tractable. In addition, the energy efficiency maximization is considered to be a fractional programming, where we first transform it into a subtractive form. Then, we consider the SDP-based SCA algorithm to recast it into the convex optimization problem with iterations. Moreover, the tightness of the SDP relaxed solution is characterized in the formulated problems. In order to gain more insights, we present a low-complexity resource allocation scheme to construct a transmit beamforming vector to reduce the computational complexity of the proposed scheme. 


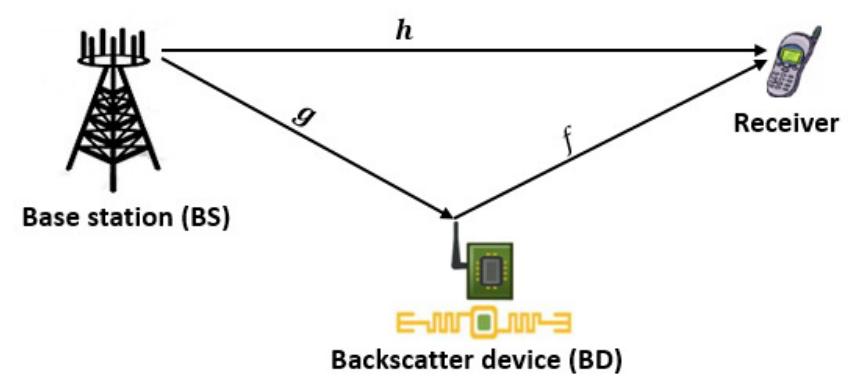

Fig. 1: A MISO SR system

The rest of this paper is organized as follows. The SR system model is first described in Section II. The problem formulation and optimal resource allocations are investigated in Section III, followed by the low complexity resource allocation in Section IV. Numerical results are presented in Section V, and we conclude this paper in Section VI.

\section{SySTEM MOdEL}

In this paper, we consider a classic BD aided MISO downlink SR as shown in Fig. 1, where it consists of a BS equipped with $N_{T} \geq 1$ transmit antenna, a BD and a receiver equipped with single-antenna, respectively. The BS establishes a direct link with the receiver and the BD intelligently adjusts its reflection coefficient via modulating its own information to carry the intended signal of the BS to the receiver. ${ }^{1}$ We consider the flat-fading channel models. Specifically, the coefficients of the channel between the BS and the receiver, which is known as the direct link, is denoted as $\mathbf{h} \in \mathbb{C}^{N_{T} \times 1}$. While, the backscatter link (i.e., $f * \mathbf{g}$ ) is the multiplication of two links: the BS and the BD (i.e., forward link), as well as the $\mathrm{BD}$ and the receiver (i.e., backward link), which are denoted by $\mathbf{g} \in \mathbb{C}^{N_{T} \times 1}$, and $f \in \mathbb{C}$, respectively. The perfect CSI of direct and backscatter links is assumed to be available at the BS and the receiver, respectively. Practically, we can employ a two-step training-based channel estimation to obtain the CSI [2]. Specifically, the BD first turns its impedance into the matched state, where the BS adopts the channel reciprocity for the channel estimation of $\mathbf{h}$. Then, the BD places its impedance into the backscatter state, where the backscatter channel $f * \mathrm{~g}$ can be obtained via extracting the estimated direct channel $\mathbf{h}$ from the the estimated composite channel $\mathbf{h}+f * \mathbf{g}$ [2]. In the $n$-th symbol period, we denote $s_{u}(n) \sim \mathcal{C N}(0,1)$ as the transmitted signal from the BS during symbol period $T_{u}$. While $s_{b}(n) \sim \mathcal{C N}(0,1)$ is denoted as the transmitted signal from the BD during symbol period $T_{b}$,

\footnotetext{
${ }^{1}$ The receiver has a cooperative role to intelligently evolves the traditional low power network infrastructure to support the passive IoT connections [2]. Smart home and smart healthcare applications are the practical scenarios of our system model [2]. Specifically, a WiFi access point (AP) at a smart home connects to a smart phone, while the IoT devices (i.e., BDs) send their own information to the smart phone via backscattering the WiFi signal, and thus the smart phone can recover information from the WiFi as well as IoT signals. In the smart healthcare scenario, the cellular BS or WiFi AP sends the messages to the wearable device (i.e., the medical sensor), and the IoT sensors backscatter the direct signal transmitted from the cellular BS or WiFi AP via modulating their own information, the wearable device can decode the messages from both the cellular BS or WiFi AP as well as the IoT devices.
}

which is adjusted according to different reflection coefficients. Thus, the backscatter signal is written as $\theta^{\frac{1}{2}} s_{b}(n)$, where $\theta \in[0,1]$ is the power reflection coefficient to control the power portion of the backscattered signal transmitted by the $\mathrm{BD}$. The transmit beamforming vector of the BS can be denoted by $\mathbf{w} \in \mathbb{C}^{N_{T} \times 1}$. The received signal at the receiver, in the $n$-th symbol period, can be written as

$$
y_{u}=\mathbf{h}^{H} \mathbf{w} s_{u}(n)+\sqrt{\theta} s_{b}(n) f \mathbf{g}^{H} \mathbf{w} s_{u}(n)+n_{u}(n),
$$

where $n_{u}(n)$ is the additive white Gaussian noise (AWGN) with zero mean and variance $\sigma_{u}^{2}$.

Remark 1: The direct link signal is stronger than the backscatter link signal, which can be explained by the facts that [2]: 1) the backscatter link goes through two attenuations, e.g., the forward link channel $g$ and the backward link channel $f ; 2)$ the BD backscatters its own signal that may also suffer from a power loss due to its reflection operation. In addition, the backscattered signal packets should be designed to be very short such that the achievable rate and block error probability can be accurately and practically measured. Thus, the backscatter link in this SR system falls into the finite blocklength channel codes.

In the following, we investigate two different SR policies based on different decoding schemes at the receiver: 1) noncooperative SR (NSR), where the receiver first decodes the direct link signal $s_{u}(n)$ which considers the BD's signal as the interference. Then the receiver decodes the BD's signal $s_{b}(n)$ in the finite block-length regime which employs the SIC to remove the estimated primary signal; 2) cooperative SR (CSR), where the receive first decodes the direct link signal $s_{u}(n)$ which treats the BD's signal as the another multipath component. Then, the receiver employs the SIC to remove direct link signal such that the BD's signal is decoded and measured in finite block-length regime.

\section{A. System Models}

1) NSR: In this subsection, we study the NSR system, where the receiver firstly decodes $s_{u}(n)$ while treating $s_{b}(n)$ as the interference. Both direct and backscatter links have the same symbol duration (i.e., $T_{u}=T_{b}$ ) such that they interfere with each other. Thus, the achievable data rate of the direct link is written as

$$
r_{u}^{\mathrm{NSR}}=\log _{2}\left(1+\gamma_{u}^{\mathrm{NSR}}\right),
$$

where $\gamma_{u}^{\text {NSR }}=\frac{\left|\mathbf{h}^{H} \mathbf{w}\right|^{2}}{\theta f\left|\mathbf{g}^{H} \mathbf{w}\right|^{2}+\sigma_{u}^{2}}$. Due to the sophisticated distribution of $s_{b}(n) s_{u}(n)$, we approximate $s_{b}(n) s_{u}(n)$ as circular symmetric complex Gaussian (CSCG) distribution ${ }^{2}$, which leads to a lower bound of the achievable rate to decode the direct link signal. After decoding the direct signal $s_{u}(n)$, the receiver decodes the BD's signal $s_{b}(n)$ via the SIC technique assuming that the direct signal $s_{u}(n)$ is cancelled perfectly [2]. Thus, the achievable data rate of the backscatter link is

\footnotetext{
${ }^{2}$ The worst-case interference can be introduced in terms of the CSCG distribution [2].
} 
written with finite block-length channel codes, which is given by [21]

$r_{b}^{\mathrm{NSR}}=(1-\epsilon)\left[\log _{2}\left(1+\gamma_{b}^{\mathrm{NSR}}\right)-\beta \sqrt{\left(1-\frac{1}{\left(1+\gamma_{b}^{\mathrm{NSR}}\right)^{2}}\right)}\right]$

where $\beta=\frac{Q^{-1}(\epsilon) \log e}{\sqrt{n}}, Q^{-1}(x)$ is the inverse of Gaussian Q-function with $Q(x)=\int_{x}^{\infty} \frac{1}{2 \pi} \exp \left(-\frac{t^{2}}{2}\right) d t, n$ denotes the length of finite block-length codebook, $\epsilon$ is the block error probability, i.e., $0<\epsilon<1$, and $\gamma_{b}^{\mathrm{NSR}}=\frac{\theta f\left|\mathbf{g}^{H} \mathbf{w} s_{u}(n)\right|^{2}}{\sigma_{u}^{2}}$. From (3), the direct link signal $s_{u}(n)$ follow CSCG distribution, thus, $\left|s_{u}(n)\right|^{2}$ follows an exponential distribution, and its probability density function (PDF) is given by $f(x)=$ $\exp (-x), x>0$. The average data rate of the backscatter link can be further written as

$\tilde{r}_{b}^{\mathrm{NSR}}=(1-\epsilon) \mathbb{E}_{s_{u}}\left[\log _{2}\left(1+\gamma_{b}^{\mathrm{NSR}}\right)-\beta \sqrt{\left(1-\frac{1}{\left(1+\gamma_{b}^{\mathrm{NSR}}\right)^{2}}\right)}\right]$.

Lemma 1: The achievable data rate of the backscatter link (4) is a monotonically increasing function in terms of $\gamma_{b}^{\mathrm{NSR}}$.

Proof: Refer to [26].

Theorem 1: A closed-form expression can be derived for the average data rate of the backscatter link, which is given by

$$
\begin{aligned}
\tilde{r}_{b}^{\mathrm{NSR}} & =(1-\epsilon)\left\{\frac{1}{\ln 2} \exp \left(\frac{1}{\tilde{\gamma}_{b}^{\mathrm{NSR}}}\right) E_{1}\left(\frac{1}{\tilde{\gamma}_{b}^{\mathrm{NSR}}}\right)-1\right. \\
+ & \left.\frac{1}{2} \frac{1}{\tilde{\gamma}_{b}^{\mathrm{NSR}}}\left[-\frac{1}{\tilde{\gamma}_{b}^{\mathrm{NSR}}} \exp \left(\frac{1}{\tilde{\gamma}_{b}^{\mathrm{NSR}}}\right) E_{1}\left(\frac{1}{\tilde{\gamma}_{b}^{\mathrm{NSR}}}\right)+1\right]\right\},
\end{aligned}
$$

where $\tilde{\gamma}_{b}^{\mathrm{NSR}}=\frac{\theta f\left|\mathbf{g}^{H} \mathbf{w}\right|^{2}}{\sigma_{u}^{2}}$.

Proof: First, we rewrite the average rate of the backscatter link as

$$
\begin{aligned}
& \tilde{r}_{b}^{\mathrm{NSR}}=(1-\epsilon)\{\underbrace{\mathbb{E}_{s_{u}}\left[\log _{2}\left(1+\bar{\gamma}_{b}^{\mathrm{NSR}} x\right)\right]}_{C} \\
& \underbrace{-\beta \mathbb{E}_{s_{u}}\left[\sqrt{\left(1-\frac{1}{\left(1+\tilde{\gamma}_{b}^{\mathrm{NSR}} x\right)^{2}}\right)}\right]}_{D}\},
\end{aligned}
$$

where $x=\left|s_{u}(n)\right|^{2}, \tilde{\gamma}_{b}^{\mathrm{NSR}}=\frac{\theta f\left|\mathbf{g}^{H} \mathbf{w}\right|^{2}}{\sigma_{u}^{2}}$. To proceed, we firstly derive the closed-form expression of $C$ from (6), which is easily given by

$$
\begin{aligned}
C & =\frac{1}{\ln 2} \int_{0}^{\infty} \exp (-x) \ln \left(1+\tilde{\gamma}_{b}^{\mathrm{NSR}} x\right) d x \\
& =\frac{1}{\ln 2} \exp \left(\frac{1}{\tilde{\gamma}_{b}^{\mathrm{NSR}}}\right) E_{1}\left(\frac{1}{\tilde{\gamma}_{b}^{\mathrm{NSR}}}\right) .
\end{aligned}
$$

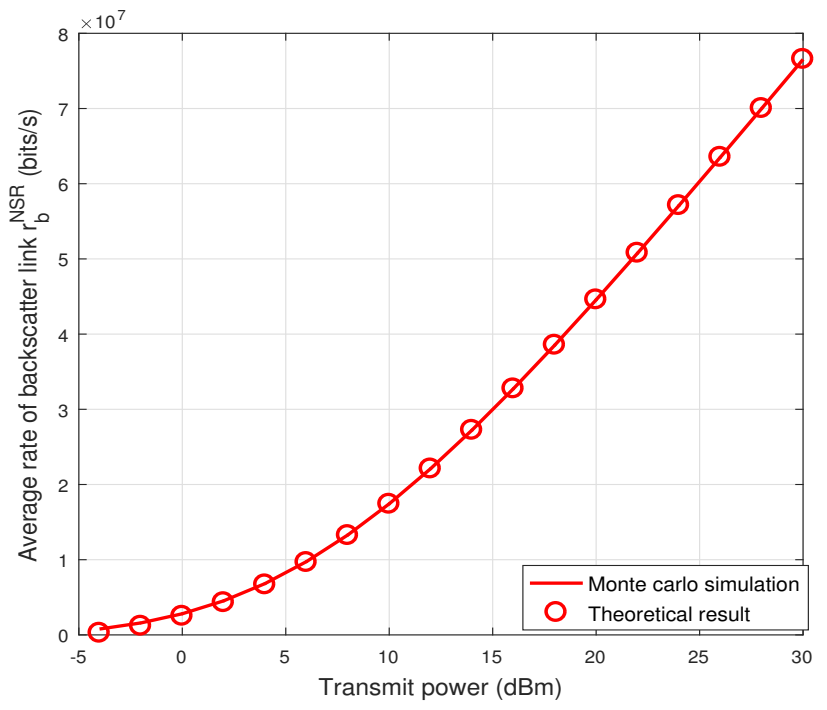

Fig. 2: Performance analysis for average rate of backscatter link in the NSR system.

We then focus on the derivation of closed-form expression for $D$, which can be expressed as

$$
D=\int_{0}^{\infty} \sqrt{\left(1-\frac{1}{\left(1+\tilde{\gamma}_{b}^{\mathrm{NSR}} x\right)^{2}}\right)} \exp (-x) d x
$$

From (8), it is observed that the closed-form expression of the integral of the term $\sqrt{1-a}$ is difficult to obtain. Thus, we consider an approximation $\sqrt{1-a} \approx 1-\frac{1}{2} a$, for $|a|<1$. By applying this approximation, we have

$$
\begin{aligned}
D & \approx \int_{0}^{\infty} \exp (-x)\left(1-\frac{1}{2} \frac{1}{\left(1+\tilde{\gamma}_{b}^{\mathrm{NSR}} x\right)^{2}}\right) d x \\
& =1-\frac{1}{2} \int_{0}^{\infty} \exp (-x) \frac{1}{\left(1+\tilde{\gamma}_{b}^{\mathrm{NSR}} x\right)^{2}} d x \\
& =1-\frac{1}{2} \frac{1}{\tilde{\gamma}_{b}^{\mathrm{NSR}}}\left[-\frac{1}{\tilde{\gamma}_{b}^{\mathrm{NSR}}} \exp \left(\frac{1}{\tilde{\gamma}_{b}^{\mathrm{NSR}}}\right) E_{1}\left(\frac{1}{\tilde{\gamma}_{b}^{\mathrm{NSR}}}\right)+1\right] .
\end{aligned}
$$

From (7) and (9), the closed-form expression of the average backscatter rate is given by (5).

We plot Fig. 2 to verify the accuracy of the proposed closedform expression of the backscatter average rate $r_{b}^{\mathrm{NRS}}$, where one can observe that the closed-form expression derived in Theorem 1 matches the Monte Carlo simulation results when the transmit power is greater than $0 \mathrm{dBm}$, which validates the effectiveness and accuracy of the approximation $\sqrt{1-a} \approx$ $1-\frac{1}{2} a$, for $|a|<1$.

2) CSR: In this subsection, we investigate the CSR system, in which the backscatter signal $s_{b}(n)$ has a longer symbol duration than $s_{u}(n)$, i.e., $T_{b}=L T_{u}$, where $L \gg 1$ is the spreading factor. Unlike the NSR system, the backscatter link signal $s_{b}(n)$ in the CSR system is transmitted during a symbol interval, which spans $L$ direct link symbol durations [2]. In the CSR system, the receiver first jointly decodes the direct and backscatter links via a multipath decoder. For given $s_{b}(n)$ 
to decode $s_{u}(n)$, the achievable data rate of the direct link can be written as

$$
r_{u}^{\mathrm{CSR}}=\log _{2}\left(1+\gamma_{u}^{\mathrm{CSR}}\right),
$$

where $\gamma_{u}^{\mathrm{CSR}}=\frac{\left|\left(\mathbf{h}+\sqrt{\theta} s_{b}(n) f \mathbf{g}\right)^{H} \mathbf{w}\right|^{2}}{\sigma_{u}^{2}}$. In this scenario, the BS is required to transmit a training symbol which estimates the multipath channel term $\mathbf{h}+\sqrt{\theta} s_{b}(n) f \mathbf{g}$ due to no prior knowledge of $s_{b}(n)$. The training symbol can be ignored in each BD symbol period with sufficient large $L$ [2]. Thus, the average rate of direct link is given by

$$
\tilde{r}_{u}^{\mathrm{CSR}}=\mathbb{E}_{s_{b}} \log _{2}\left[\left(1+\gamma_{u}^{\mathrm{CSR}}\right)\right] .
$$

We denote $z=\frac{\left|\left(\mathbf{h}+\sqrt{\theta} s_{b}(n) f \mathbf{g}\right)^{H} \mathbf{w}\right|^{2}}{\sigma_{u}^{2}}$, which follows the noncentral chi-square distribution two degrees of freedom [2], [15]. Its PDF is given by

$f_{z}(x)=\frac{\sigma_{u}^{2}}{\theta|f|^{2}\left|\mathbf{g}^{H} \mathbf{w}\right|^{2}} \exp \left(-\frac{x+\frac{\left|\mathbf{h}^{H} \mathbf{w}\right|^{2}}{\sigma_{u}^{2}}}{\frac{\theta|f|^{2}\left|\mathbf{g}^{H} \mathbf{w}\right|^{2}}{\sigma_{u}^{2}}}\right) I_{0}\left(\frac{\left(\frac{\left|\mathbf{h}^{H} \mathbf{w}\right|^{2}}{\sigma_{u}^{2}} x\right)^{\frac{1}{2}}}{\frac{\theta|f|^{2}\left|\mathbf{g}^{H} \mathbf{w}\right|^{2}}{2 \sigma_{u}^{2}}}\right)$,

where $I_{0}(x)=\sum_{n=0}^{\infty} \frac{1}{n ! \Gamma(n+1)}\left(\frac{x}{2}\right)^{2 n}$ denotes a modified Bessel function of the first kind. Thus, the average rate (11) can be equivalently modified as

$$
\tilde{r}_{u}^{\mathrm{CSR}}=\mathbb{E}_{z}\left[\log _{2}(1+z)\right]=\int_{0}^{\infty} \log _{2}(1+x) f_{z}(x) d x .
$$

From (13), it is intractable to derive the closed-form expression with its complicated integrals. Thus, we consider an asymptotic approximation at the high SNR regime. When, the SNR is sufficient large, we have the closed-form expression for the average rate $r_{u}$ as [2], [15]

$$
\tilde{r}_{u}^{\mathrm{CSR}} \approx \log _{2}\left(\frac{\left|\mathbf{h}^{H} \mathbf{w}\right|^{2}}{\sigma_{u}^{2}}\right)+\frac{1}{\ln 2} E_{1}\left(\frac{\left|\mathbf{h}^{H} \mathbf{w}\right|^{2}}{\theta|f|^{2}\left|\mathbf{g}^{H} \mathbf{w}\right|^{2}}\right) .
$$

We plot Fig. 3 to show the average rate of direct link $r_{u}^{\mathrm{CSR}}$ The accuracy of (14) is presented in Fig. 3. It is observed from this figure that there is a gap between the closed-form expression in (14) and the Monte Carlo simulation in the low transmit power region. As the transmit power increases, the gap becomes smaller and smaller such that the closed-form expression achieves the same performance with the Monte Carlo simulation with sufficient large transmit power.

Then, the signal of the backscatter link $s_{b}$ will be decoded for given $s_{u}(n)$ which is based on the assumption that the direct link term has been removed perfectly via SIC. Thus, the received signal can be modified in the vector form during the BD symbol duration $T_{b}$ which is given by

$$
\mathbf{y}_{u}=\sqrt{\theta} s_{b} f \mathbf{g}^{H} \mathbf{w} \mathbf{s}_{u}+\mathbf{n}_{u},
$$

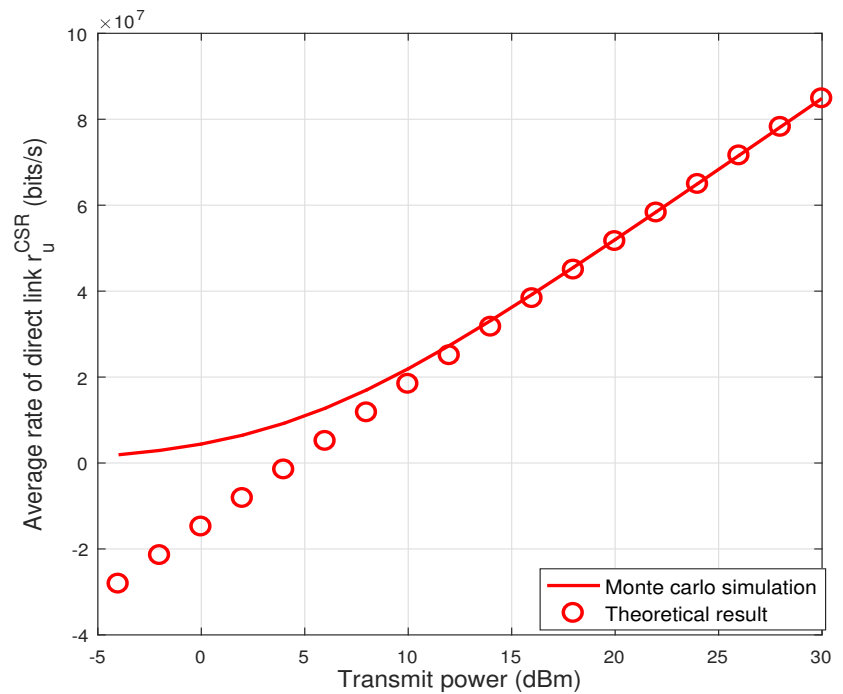

Fig. 3: Performance analysis for average rate of direct link in the CSR system.

where $\mathbf{y}_{u}=\left[y_{u}(1), \ldots, y_{u}(L)\right], \mathbf{s}_{u}=\left[s_{u}(1), \ldots, s_{u}(L)\right]$, and $\mathbf{n}_{u}=\left[n_{u}(1), \ldots, n_{u}(L)\right]$. By exploiting the maximal ratio combining (MRC) and finite block-length channel codes,

$$
\begin{aligned}
r_{c}^{\mathrm{CSR}} & =\frac{1}{L} \mathbb{E}_{\mathbf{s}_{u}}\left\{\operatorname { l o g } _ { 2 } \left[\left[1+\frac{\sum_{n=1}^{N} \theta|f|^{2}\left|\mathbf{g}^{H} \mathbf{w}\right|^{2}\left|s_{u}(n)\right|^{2}}{\sigma_{u}^{2}}\right]\right.\right. \\
& -\beta \sqrt{\left.\left(1-\frac{1}{\left(1+\frac{\sum_{n=1}^{N} \theta|f|^{2}\left|\mathbf{g}^{H} \mathbf{w}\right|^{2}\left|s_{u}(n)\right|^{2}}{\sigma_{u}^{2}}\right)^{2}}\right)\right]},
\end{aligned}
$$

By applying $\mathbb{E}\left\{\left|s_{u}(n)\right|^{2}\right\}=1$,

$$
r_{c}^{\mathrm{CSR}}=\frac{1}{L} \log _{2}\left[\left(1+\gamma_{b}^{\mathrm{CSR}}\right)-\beta \sqrt{\left(1-\frac{1}{\left(1+\gamma_{b}^{\mathrm{CSR}}\right)^{2}}\right)}\right],
$$

where $\gamma_{b}^{\mathrm{CSR}}=\frac{L \theta|f|^{2}\left|\mathbf{g}^{H} \mathbf{w}\right|^{2}}{\sigma_{u}^{2}}$.

\section{B. Problem formulation}

By exploiting the two SR systems in Section II-A, we formulate the following optimization problems: 1) transmit power minimization problem; 2) energy efficiency maximization problem, to achieve the optimal resource allocation.

1) Transmit Power Minimization: The transmit power minimization problem is formulated subject to the achievable throughput of the direct and backscatter links.

1) NSR: We formulate the transmit power minimization problem for the NSR system as follows:

$$
\begin{gathered}
\min _{\mathbf{w}}\|\mathbf{w}\|^{2}, \\
\text { s.t. } B \log _{2}\left(1+\gamma_{u}^{\mathrm{NSR}}\right) \geq R_{u}, \\
B(1-\epsilon) \mathbb{E}_{s_{u}}\left[\log _{2}\left(1+\gamma_{b}^{\mathrm{NSR}}\right)-\beta \sqrt{\left(1-\frac{1}{\left(1+\gamma_{b}^{\mathrm{NSR}}\right)^{2}}\right)}\right] \\
\geq \frac{S}{T},
\end{gathered}
$$


where $\beta=\frac{Q^{-1}(\epsilon) \log e}{\sqrt{n}}, R_{u}$ is the target rate of the direct link, $S$ denotes the packet size received from the backscatter link at the receiver, $B$ is the total system bandwidth, and $T$ is the maximum packet delay threshold.

2) $C S R$ : We formulate the transmit power minimization problem for the CSR system as follows:

$$
\begin{aligned}
& \min _{\mathbf{w}}\|\mathbf{w}\|^{2} \\
& \text { s.t. } B \mathbb{E}_{s_{b}}\left[\log _{2}\left(1+\gamma_{u}^{\mathrm{CSR}}\right)\right] \geq R_{u} \\
& \frac{B(1-\epsilon)}{L} \log _{2}\left[\left(1+\gamma_{b}^{\mathrm{CSR}}\right)-\beta \sqrt{\left.\left(1-\frac{1}{\left(1+\gamma_{b}^{\mathrm{CSR}}\right)^{2}}\right)\right]}\right. \\
& \geq \frac{S}{T} .
\end{aligned}
$$

2) Energy Efficiency Maximization: We formulate the energy efficiency maximization problem subject to the achievable throughput requirement for the direct and backscatter links as well as the transmit power constraint.

1) NSR: We formulate the energy efficiency maximization problem for the NSR system as (20) on the top of next page, where $P_{\max }$ is the maximum transmit power at the $\mathrm{BS}$, and $\xi$ is a constant which denotes the inefficiency of power amplifier [27].

2) CSR: We formulate the energy efficiency maximization problem for the CSR system as (21) on the top of next page,

It is verified that the transmit power minimization problems in Section II-B1 and the energy efficiency maximization problems in Section II-B2 are not convex, which cannot be solved directly. In this following we will develop a SDP relaxation and a SCA approach to iteratively handle these non-convex optimization problems.

\section{Optimal Resource Allocation}

In order to solve the formulated problems in Section II-B, we propose a SDP based relaxation approach to solve the transmit power minimization and the energy efficiency maximization problems, respectively.

\section{A. Transmit Power Minimization}

In this subsection, we employ the SDP relaxation to solve the transmit power minimization problem for the NSR and CSR systems.

1) NSR: We define $\mathbf{W}=\mathbf{w} \mathbf{w}^{H}$, and substitute the closedform expression in Theorem 1 into (18b), problem (18) can be rewritten as

$$
\begin{aligned}
& \min _{\mathbf{W} \succeq \mathbf{0}} \operatorname{Tr}(\mathbf{W}), \text { s.t. } \operatorname{rank}(\mathbf{W})=1, \\
& B \log _{2}\left(1+\frac{\operatorname{Tr}\left(\mathbf{h} \mathbf{h}^{H} \mathbf{W}\right)}{\theta f \operatorname{Tr}\left(\mathbf{g g}^{H} \mathbf{W}\right)+\sigma_{u}^{2}}\right) \geq R_{u}, \\
& B(1-\epsilon)\left\{\frac{1}{\ln 2} \exp \left(\frac{\sigma_{u}^{2}}{\theta f \operatorname{Tr}\left(\mathbf{g g}^{H} \mathbf{W}\right)}\right) E_{1}\left(\frac{\sigma_{u}^{2}}{\theta f \operatorname{Tr}\left(\mathbf{g g}^{H} \mathbf{W}\right)}\right)\right. \\
& -\beta+\frac{\sigma_{u}^{2} \beta}{2 \theta f \operatorname{Tr}\left(\mathbf{g g}^{H} \mathbf{W}\right)}\left[-\frac{\sigma_{u}^{2}}{\theta f \operatorname{Tr}\left(\mathbf{g g}^{H} \mathbf{W}\right)}\right. \\
& \left.\left.\quad * \exp \left(\frac{\sigma_{u}^{2}}{\theta f \operatorname{Tr}\left(\mathbf{g g}^{H} \mathbf{W}\right)}\right) E_{1}\left(\frac{\sigma_{u}^{2}}{\theta f \operatorname{Tr}\left(\mathbf{g g}^{H} \mathbf{W}\right)}\right)+1\right]\right\} \geq \frac{S}{T},
\end{aligned}
$$

Problem (22) is still non-convex and intractable in terms of the non-convex rank-one constraint and constraint (22b). To procceed, we adopt the SDP relaxation to remove the non-convex rank-one constraint and equivalently modified the achievable rate constraint of the backscatter link, which is given by

$$
\begin{array}{ll}
\min _{\mathbf{W} \succeq \mathbf{0}} \operatorname{Tr}(\mathbf{W}), \\
\text { s.t. } \operatorname{Tr}\left(\mathbf{h} \mathbf{h}^{H} \mathbf{W}\right)-\left(2^{\frac{R_{u}}{B}}-1\right) \theta f \operatorname{Tr}\left(\mathbf{g g}^{H} \mathbf{W}\right) \\
\quad-\left(2^{\frac{R_{u}}{B}}-1\right) \sigma_{u}^{2} \geq 0, \\
\frac{\theta f \operatorname{Tr}\left(\mathbf{g g}^{H} \mathbf{W}\right)}{\sigma_{u}^{2}} \geq \Upsilon_{\tilde{x}}\left(\frac{S}{T}\right),
\end{array}
$$

where $\Upsilon_{\tilde{x}}\left(\frac{S}{T}\right)$ is the root of the following equation:

$$
\begin{aligned}
& B(1-\epsilon)\left\{\frac{1}{\ln 2} \exp \left(\frac{1}{\tilde{x}}\right) E_{1}\left(\frac{1}{\tilde{x}}\right)\right. \\
& \left.-\beta+\frac{\beta}{2 \tilde{x}}\left[-\frac{1}{\tilde{x}} \exp \left(\frac{1}{\tilde{x}}\right) E_{1}\left(\frac{1}{\tilde{x}}\right)+1\right]\right\}=\frac{S}{T}
\end{aligned}
$$

It is easily observed that problem (23) is a standard SDP, which can be solved via interior-point method [28]. We first examine the tightness of the SDP relaxed solution to problem (23). If the optimal solution to (23) (denoted by $\mathbf{W}^{*}$ ) is a rank-one matrix, the transmit beamforming vector can be achieved directly via the eigenvalue decomposition. Otherwise, we investigate a construction method to achieve a rank-one solution. Particularly, upon applying the eigenvalue decomposition to $\mathbf{W}^{*}$, i.e., $\mathbf{W}^{*}=\mathbf{U} \Theta \mathbf{U}^{\mathbf{H}}$, and the feasible beamforming vector to (18) $\tilde{\mathbf{w}}$ is constructed as

$$
\tilde{\mathbf{w}}=\mathbf{U} \Theta^{\frac{1}{2}} \mathbf{r}
$$

where $\mathbf{U} \in \mathbb{C}^{N_{T} \times N_{T}}$ is a unitary matrix, $\Theta \in \mathbb{C}^{N_{T} \times N_{T}}$ denotes a diagonal matrix with eigenvalues arranged in decreasing order, and $\mathbf{r}$ is a vector of complex circularly symmetric uncorrelated Gaussian random variables with zero-mean and unit variance. However, problem (23) may not guarantee its constraints with the independent generation of Gaussian random vector $\mathbf{r}$. We thus re-scale the vector $\mathbf{r}$ with an appropriate scaling factor $\varrho$ to satisfy all constraints, as

$$
\overline{\mathbf{w}}=\varrho \tilde{\mathbf{w}},
$$




$$
\begin{aligned}
\max _{\mathbf{w}} \frac{B \log _{2}\left(1+\gamma_{u}^{\mathrm{NSR}}\right)+B(1-\epsilon) \mathbb{E}_{s_{u}}\left[\log _{2}\left(1+\gamma_{b}^{\mathrm{NSR}}\right)-\beta \sqrt{\left(1-\frac{1}{\left(1+\gamma_{b}^{\mathrm{NSR}}\right)^{2}}\right)}\right.}{\xi\|\mathbf{w}\|^{2}+P_{c}}, \\
\text { s.t. }\|\mathbf{w}\|^{2} \leq P_{\max }, \\
\quad B \log _{2}\left(1+\gamma_{u}^{\mathrm{NSR}}\right) \geq R_{u}, \\
\quad B(1-\epsilon) \mathbb{E}_{s_{u}}\left[\log _{2}\left(1+\gamma_{b}^{\mathrm{NSR}}\right)-\beta \sqrt{\left.\left(1-\frac{1}{\left(1+\gamma_{b}^{\mathrm{NSR}}\right)^{2}}\right)\right] \geq \frac{S}{T}}\right.
\end{aligned}
$$

$$
\begin{aligned}
& \max _{\mathbf{w}} \frac{B \mathbb{E}_{s_{b}}\left(1+\gamma_{u}^{\mathrm{CSR}}\right)+\frac{B(1-\epsilon)}{L} \log _{2}\left[\left(1+\gamma_{b}^{\mathrm{CSR}}\right)-\beta \sqrt{\left(1-\frac{1}{\left(1+\gamma_{b}^{\mathrm{CSR}}\right)^{2}}\right)}\right]}{\xi\|\mathbf{w}\|^{2}+P_{c}}, \\
& \text { s.t. }\|\mathbf{w}\|^{2} \leq P_{\max }, \\
& B \mathbb{E}_{s_{b}} \log _{2}\left[\left(1+\gamma_{u}^{\mathrm{CSR}}\right)\right] \geq R_{u}, \\
& \frac{B(1-\epsilon)}{L} \log _{2}\left[\left(1+\gamma_{b}^{\mathrm{CSR}}\right)-\beta \sqrt{\left(1-\frac{1}{\left(1+\gamma_{b}^{\mathrm{CSR}}\right)^{2}}\right)}\right] \geq \frac{S}{T} \text {. }
\end{aligned}
$$

We substitute (26) into (23) to obtain the optimal scaling factor via solving the following problem:

$$
\begin{aligned}
& \max _{\varpi} \operatorname{Tr}(\hat{\mathbf{W}}) \\
& \text { s.t. } \operatorname{Tr}\left(\mathbf{h} \mathbf{h}^{H} \hat{\mathbf{W}}\right)-\left(2^{\frac{R_{u}}{B}}-1\right) \theta f \operatorname{Tr}\left(\mathbf{g g}^{H} \hat{\mathbf{W}}\right)-\left(2^{\frac{R_{u}}{B}}-1\right) \sigma_{u}^{2} \geq 0, \\
& \qquad \theta f \operatorname{Tr}\left(\mathbf{g g}^{H} \hat{\mathbf{W}}\right) \geq \Upsilon_{\tilde{x}}\left(\frac{S}{T}\right) \sigma_{u}^{2}
\end{aligned}
$$

where $\hat{\mathbf{W}}=\varpi \tilde{\mathbf{w}} \tilde{\mathbf{w}}^{H}, \varpi=\varrho^{2}$. It can be easily shown that problem (27) is a standard linear programming (LP), which is easily solved. By exploiting the beamforming vector scaling, the rank-one optimal solution finally can be achieved via (25) and (26).

2) CSR: We first consider the SDP to relax problem (19), i.e., $\mathbf{W}=\mathbf{w} \mathbf{w}^{H}$, which is given by

$$
\begin{aligned}
& \min _{\mathbf{W} \succeq \mathbf{0}} \operatorname{Tr}(\mathbf{W}), \text { s.t. } \operatorname{rank}(\mathbf{W})=1, \\
& B \mathbb{E}_{s_{b}}\left[\log _{2}\left(1+\frac{\operatorname{Tr}\left(\overline{\mathbf{h}} \overline{\mathbf{h}}^{H} \mathbf{W}\right)}{\sigma_{u}^{2}}\right)\right] \geq R_{u}, \\
& \frac{B(1-\epsilon)}{L} \log _{2}\left[\left(1+\frac{L \theta|f|^{2} \operatorname{Tr}\left(\mathbf{g g}^{H} \mathbf{W}\right)}{\sigma_{u}^{2}}\right)\right. \\
& -\beta \sqrt{\left(1-\frac{1}{\left(1+\frac{L \theta|f|^{2} \operatorname{Tr}\left(\mathbf{g g}^{H} \mathbf{W}\right)}{\sigma_{u}^{2}}\right)^{2}}\right)} \geq \frac{S}{T} .
\end{aligned}
$$

where $\overline{\mathbf{h}}=\mathbf{h}+\sqrt{\theta} s_{b}(n) f \mathbf{g}$. Problem (28) is still non-convex and intractable due to the non-convex rank-one constraint and the constraint (28b). In order to circumvent this nonconvex issue, we first consider the channel dispersion term $R=1-\frac{1}{\left(1+\gamma_{b}^{\mathrm{CSR}}\right)^{2}}$. It is verified that $\sqrt{R}$ is a concave function in terms of $\gamma_{b}^{\mathrm{CSR}}>0$ such that (17) is easily verified that difference of concave (DC) programming.. In order to tackle the constraint (28b), we consider SCA to linearize the channel dispersion term in (28b). SCA has been considered as one of the efficient approaches to solve the DC programming problems. The main idea is that the original non-convex problem is convexified with a locally tight approximation, which iteratively produces a tight convex objective function and constraint sets. It means that a series of convex optimization problem is solved to achieve an approximated solution rather than solving the original non-convex optimization problem directly. Accordingly, we consider [29, Assumption 1] to adopt the SCA to approximate the constraint (28b). By exploiting the SCA, the channel dispersion term can be approximated at the $i$ iteration, as (29) on the top of the next page. It is verified that the approximation (29) satisfies [29, Assumption 1]. Thus, the constraint (28b) can be reformulated as (30) on the top of the next page. By removing the non-convex rank-one constraint and replacing (22b) with (30), problem (28) is reformulated at the $i$-th iteration, as

$$
\begin{aligned}
& \min _{\mathbf{W} \succeq \mathbf{0}} \operatorname{Tr}(\mathbf{W}), \\
& \text { s.t. } B \mathbb{E}_{s_{b}}\left[\log _{2}\left(1+\frac{\operatorname{Tr}\left(\overline{\mathbf{h}} \overline{\mathbf{h}}^{H} \mathbf{W}\right)}{\sigma_{u}^{2}}\right)\right] \geq R_{u},
\end{aligned}
$$

Problem (31) is a convex optimization problem, which can be solved via interior-point method. We summarize the SCA in Algorithm 1. Also, we provide a flowchart in Fig. 4 to show the procedure of Algorithm 1.

\section{Algorithm 1: The SCA Algorithm}

1) Input: $\kappa>0$ is the very small constant, $\mathcal{P}^{(i)}$ is the optimal objective value of problem (31) at the $i$-th iteration. Initialization: $\mathbf{W}^{(0)}$. 


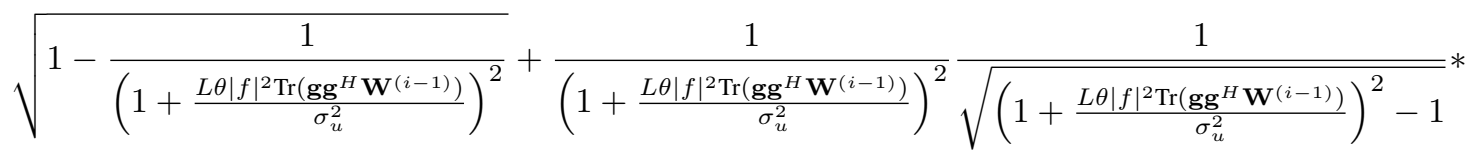

$$
\begin{aligned}
& {\left[1+\frac{L \theta|f|^{2} \operatorname{Tr}\left(\mathbf{g g}^{H} \mathbf{W}\right)}{\sigma_{u}^{2}}-\left(1+\frac{L \theta|f|^{2} \operatorname{Tr}\left(\mathbf{g g}^{H} \mathbf{W}^{(i-1)}\right)}{\sigma_{u}^{2}}\right)\right] \geq \sqrt{R}}
\end{aligned}
$$

$$
\begin{aligned}
&(28 \mathrm{~b}) \Rightarrow \frac{B(1-\epsilon)}{L}\left\{\log _{2}\left(1+\frac{L \theta|f|^{2} \operatorname{Tr}\left(\mathbf{g g}^{H} \mathbf{W}\right)}{\sigma_{u}^{2}}\right)-\beta\left[\sqrt{1-\frac{1}{\left(1+\frac{L \theta|f|^{2} \operatorname{Tr}\left(\mathbf{g g}^{H} \mathbf{W}^{(i-1)}\right)}{\sigma_{u}^{2}}\right)^{2}}}+\frac{1}{\left(1+\frac{L \theta|f|^{2} \operatorname{Tr}\left(\mathbf{g g}^{H} \mathbf{W}^{(i-1)}\right)}{\sigma_{u}^{2}}\right)^{2}} *\right.\right. \\
&\left.\left.\frac{1}{\sqrt{\left(1+\frac{L \theta|f|^{2} \operatorname{Tr}^{2}\left(\mathbf{g g}^{H} \mathbf{W}^{(i-1)}\right)}{\sigma_{u}^{2}}\right)^{2}-1}} *\left[1+\frac{L \theta|f|^{2} \operatorname{Tr}\left(\mathbf{g g}^{H} \mathbf{W}\right)}{\sigma_{u}^{2}}-\left(1+\frac{L \theta|f|^{2} \operatorname{Tr}\left(\mathbf{g g}^{H} \mathbf{W}^{(i-1)}\right)}{\sigma_{u}^{2}}\right)\right]\right]\right\} \geq \frac{S}{T} .
\end{aligned}
$$

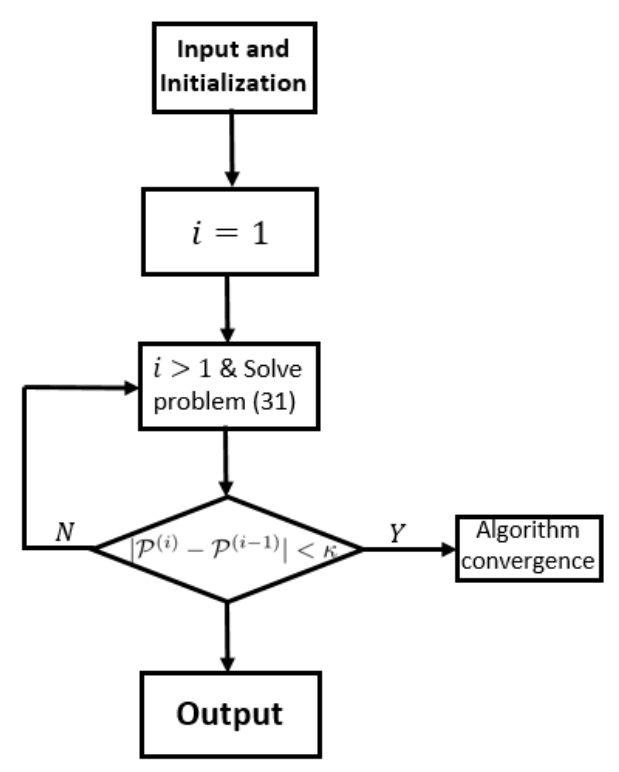

Fig. 4: Flowchart of Algorithm 1.

2) Repeat: $i>1$

a) Solve problem (31) with $\mathbf{W}^{(i-1)}$ to obtain $\mathbf{W}^{(i)}$.

b) If $\left|\mathcal{P}^{(i)}-\mathcal{P}^{(i-1)}\right|<\kappa$, then

- Problem (31) converges to the approximated solution.

c) Else

- $i=i+1$, go to Step 2-a .

\section{d) End if}

3) Output: SDP relaxed solution $\mathbf{W}^{*}=\mathbf{W}^{(i)}$.

Next, we examine the convergence property of Algorithm 1 and the tightness of the SDP relaxation for $\mathbf{W}^{*}$.

Theorem 2: Every limit point $\tilde{\mathbf{W}}$ is iteratively generated by the SCA process in Algorithm 1, converges to a Karush-KuhnTucker (KKT) point of problem (31).

Proof: Refer to [30].
In order to investigate the tightness of the SDP relaxed solution, (31) can be equivalently written as

$$
\begin{aligned}
& \min _{\mathbf{W}^{(i)} \succeq \mathbf{0}} \operatorname{Tr}\left(\mathbf{W}^{(i)}\right), \\
& \text { s.t. } \operatorname{Tr}\left(\hat{\mathbf{h}} \hat{\mathbf{h}}^{H} \mathbf{W}^{(i)}\right)-\left(2^{\frac{R_{u}}{B}}-1\right) \sigma_{u}^{2} \geq 0, \\
& \frac{B(1-\epsilon)}{L}\left[\log _{2}\left(1+\frac{L \theta|f|^{2} \operatorname{Tr}\left(\mathbf{g g}^{H} \mathbf{W}^{(i)}\right)}{\sigma_{u}^{2}}\right)\right. \\
& -\beta \sqrt{\left.1-\frac{1}{\left(1+\frac{L \theta|f|^{2} \operatorname{Tr}\left(\mathbf{g g}^{H} \mathbf{W}^{(i-1)}\right)}{\sigma_{u}^{2}}\right)^{2}}\right] \geq \frac{S}{T},}
\end{aligned}
$$

where $\hat{\mathbf{h}}=\mathbb{E}_{s_{b}}(\overline{\mathbf{h}})$. Problem (32) can be solved for given $\mathbf{W}^{(i-1)}$ to achieve the converged solution $\mathbf{W}^{(i)}$ at the $i$-th iteration, which guarantees the convergence of Algorithm 1. The remaining part is to investigate the tightness of (32) at the $i$-th iteration. Similar to Section III-A1, we consider the eigenvalue decomposition to directly achieve the optimal beamforming vector if $\operatorname{rank}\left(\mathbf{W}^{(i)}\right)=1$. Otherwise, the construction method shown in Section III-A1 can be employed to achieve the rankone solution, which is omitted here.

\section{B. Energy Efficiency Maximization}

In the subsection, we consider the resource allocation scheme for the energy efficiency maximization problem for the NSR and CSR systems, respectively.

1) NSR: First, we define $\mathbf{W}=\mathbf{w} \mathbf{w}^{H}$, and substitute (5) into (20a), (20) is given by

$$
\begin{aligned}
\max _{\mathbf{W} \succeq \mathbf{0}} \frac{B \log _{2}\left(1+\frac{\operatorname{Tr}\left(\mathbf{h h}^{H} \mathbf{W}\right)}{\theta f \operatorname{Tr}\left(\mathbf{g g}^{H} \mathbf{W}\right)+\sigma_{u}^{2}}\right)+\chi(\mathbf{W})}{\xi \operatorname{Tr}(\mathbf{W})+P_{c}}, \\
\text { s.t. } \operatorname{Tr}(\mathbf{W}) \leq P_{\max }, \\
B \log _{2}\left(1+\frac{\operatorname{Tr}\left(\mathbf{h h}^{H} \mathbf{W}\right)}{\theta f \operatorname{Tr}\left(\mathbf{g g}^{H} \mathbf{W}\right)+\sigma_{u}^{2}}\right) \geq R_{u}, \\
\chi(\mathbf{W}) \geq \frac{S}{T}, \\
\operatorname{rank}(\mathbf{W})=1 .
\end{aligned}
$$


where

$$
\begin{aligned}
\chi(\mathbf{W}) & =B(1-\epsilon)\left\{\frac{1}{\ln 2} \exp \left(\frac{\sigma_{u}^{2}}{\theta f \operatorname{Tr}\left(\mathbf{g g}^{H} \mathbf{W}\right)}\right) *\right. \\
& E_{1}\left(\frac{\sigma_{u}^{2}}{\theta f \operatorname{Tr}\left(\mathbf{g g}^{H} \mathbf{W}\right)}\right)-\beta+\frac{\sigma_{u}^{2} \beta}{2 \theta f \operatorname{Tr}\left(\mathbf{g g}^{H} \mathbf{W}\right)} \\
& *\left[-\frac{\sigma_{u}^{2}}{\theta f \operatorname{Tr}\left(\mathbf{g g}^{H} \mathbf{W}\right)} \exp \left(\frac{\sigma_{u}^{2}}{\theta f \operatorname{Tr}\left(\mathbf{g g}^{H} \mathbf{W}\right)}\right)\right. \\
& \left.\left.* E_{1}\left(\frac{\sigma_{u}^{2}}{\theta f \operatorname{Tr}\left(\mathbf{g g}^{H} \mathbf{W}\right)}\right)+1\right]\right\} .
\end{aligned}
$$

Problem (33) is still non-convex and intractable in terms of the objective function (33a), the constraints (33b), (33c), and (33d). In order to circumvent this issue, we propose a twolevel based SDP relaxation. First, we consider the outer level optimization to tackle (33a). In particularly, it is observed that (33a) belongs to a fractional programming [31], which can be transformed into the following subtractive form:

$$
\begin{gathered}
\max _{\mathbf{W} \succeq \mathbf{0}} B \log _{2}\left(1+\frac{\operatorname{Tr}\left(\mathbf{h h}^{H} \mathbf{W}\right)}{\theta f \operatorname{Tr}\left(\operatorname{gg}^{H} \mathbf{W}\right)+\sigma_{u}^{2}}\right)+\chi(\mathbf{W}) \\
-q\left(\xi \operatorname{Tr}(\mathbf{W})+P_{c}\right), \\
\text { s.t. } \operatorname{Tr}(\mathbf{W}) \leq P_{\max }, \quad(33 \mathrm{~b}), \quad(33 \mathrm{c}),
\end{gathered}
$$

where $q$ is a non-negative parameter, and its optimal value (i.e., $\left.q^{*}\right)$ is defined as maximum energy efficiency, which satisfies

$$
\begin{gathered}
\max _{\mathbf{W} \succeq \mathbf{0}}\left[B \log _{2}\left(1+\frac{\operatorname{Tr}\left(\mathbf{h h}^{H} \mathbf{W}\right)}{\theta f \operatorname{Tr}\left(\mathbf{g g}^{H} \mathbf{W}\right)+\sigma_{u}^{2}}\right)+\chi(\mathbf{W})\right. \\
\left.-q^{*}\left(\xi \operatorname{Tr}(\mathbf{W})+P_{c}\right)\right]=0 .
\end{gathered}
$$

The solution to (35) has been provided in [31], where an iterative algorithm is proposed to obtain $q$ via solving (35) at each iteration until convergence. Next, we consider the inner level to solve the subtractive problem (34) for given $q$. We introduce a variable $\bar{T}_{b}$ to replace $\chi(\mathbf{W})$,

$$
\begin{gathered}
\max _{\mathbf{W} \succeq \mathbf{0}, \bar{T}_{b}} B \log _{2}\left(1+\frac{\operatorname{Tr}\left(\mathbf{h} \mathbf{h}^{H} \mathbf{W}\right)}{\theta f \operatorname{Tr}\left(\mathbf{g g}^{H} \mathbf{W}\right)+\sigma_{u}^{2}}\right)+\bar{T}_{b} \\
-q\left(\xi \operatorname{Tr}(\mathbf{W})+P_{c}\right), \\
\text { s.t. } \operatorname{Tr}(\mathbf{W}) \leq P_{\max },(33 \mathrm{~b}) \\
\chi(\mathbf{W}) \geq \max \left\{\frac{S}{T}, \bar{T}_{b}\right\},
\end{gathered}
$$

In order to tackle the achievable rate of the direct link in (36a), we consider the SCA at the $i$-th iteration,

$$
\begin{aligned}
r_{u} \geq & \log _{2}\left(\operatorname{Tr}\left(\mathbf{h} \mathbf{h}^{H} \mathbf{W}\right)+\theta f \operatorname{Tr}\left(\mathbf{g g}^{H} \mathbf{W}\right)+\sigma_{u}^{2}\right) \\
& -\log _{2}\left(\theta f \operatorname{Tr}\left(\mathbf{g g}^{H} \mathbf{W}^{(i-1)}\right)+\sigma_{u}^{2}\right) \\
& -\frac{1}{\ln 2} \frac{1}{\theta f \operatorname{Tr}\left(\mathbf{g g}^{H} \mathbf{W}^{(i-1)}\right)+\sigma_{u}^{2}}\left[\theta f \operatorname{Tr}\left(\mathbf{g g}^{H} \mathbf{W}\right)\right. \\
& \left.-\theta f \operatorname{Tr}\left(\mathbf{g g}^{H} \mathbf{W}^{(i-1)}\right)\right] \triangleq r_{u}^{(i)} .
\end{aligned}
$$

Also, constraint (36c) can be recast via introducing a variable $t_{b}$ as

$$
\text { (36c) } \Rightarrow\left\{\begin{array}{l}
\theta f \operatorname{Tr}\left(\mathbf{g g}^{H} \mathbf{W}\right) \geq \Gamma_{b}\left(t_{b}\right) \sigma_{u}^{2}, \\
t_{b} \geq \max \left\{\frac{S}{T}, \bar{T}_{b}\right\}
\end{array}\right.
$$

where $\Gamma_{\tilde{x}}\left(t_{b}\right)$ is the root the following equations:

$$
\begin{aligned}
B(1-\epsilon) & \left\{\frac{1}{\ln 2} \exp \left(\frac{1}{\tilde{x}}\right) E_{1}\left(\frac{1}{\tilde{x}}\right)-\beta\right. \\
& \left.+\frac{\beta}{2 \tilde{x}}\left[-\frac{1}{\tilde{x}} \exp \left(\frac{1}{\tilde{x}}\right) E_{1}\left(\frac{1}{\tilde{x}}\right)+1\right]\right\}=t_{b} .
\end{aligned}
$$

At the $i$-th iteration, (36) can be transformed as

$$
\begin{array}{rl}
\max _{\mathbf{W} \succeq \mathbf{0}, \bar{T}_{b}, t_{b}} & B r_{u}^{(i)}+\bar{T}_{b}-q\left(\xi \operatorname{Tr}(\mathbf{W})+P_{c}\right), \\
\text { s.t. } & \operatorname{Tr}(\mathbf{W}) \leq P_{\text {max }},(38) \\
& \operatorname{Tr}\left(\mathbf{h} \mathbf{h}^{H} \mathbf{W}\right)-\left(2^{\frac{R_{u}}{B}}-1\right) \theta f \operatorname{Tr}\left(\mathbf{g g}^{H} \mathbf{W}\right) \\
& -\left(2^{\frac{R_{u}}{B}}-1\right) \sigma_{u}^{2} \geq 0 .
\end{array}
$$

Problem (40) is not jointly convex in terms of $\mathbf{W}, \bar{T}_{b}$, and $t_{b}$. Thus, we first solve problem (40) to obtain the optimal solution $\mathbf{W}^{*}$ and $\bar{T}_{b}^{*}$ for a given $t_{b}$, and then the optimal $t_{b}^{*}$ can be achieved via one-dimensional exhaustive search (i.e., bisection search). The solution to (33) is summarized in Algorithm 2. Also, we provide a flowchart in Fig. 5 to show the procedure of Algorithm 2.

Algorithm 2: Solving energy efficiency maximization problem (33)

1) Input: $(\kappa, \rho)>0$ are very small constants, $\mathcal{Q}^{(i)}$ is the optimal objective value of (40) at the $i$-th iteration, and $q^{(j)}$ is the optimal value of (33) at the $j$-th iteration. Initialization: $\mathbf{W}^{(0)}$ and $q^{(0)}$

2) Outer level repeat: $j>1$

a) Inner level repeat: $i>1$

i) Solve problem (40) for a given $t_{b}$ to obtain $\mathbf{W}^{(i)}$ and $\bar{T}_{b}^{(i)}$.

ii) If $\left|\mathcal{Q}^{(i)}-\mathcal{Q}^{(i-1)}\right|<\kappa$, then

- Problem (40) converges to the approximated solution.

iii) Else

- i $=$ i+1, go to Step 2-a-i.

b) Obtain the optimal solution $t_{b}^{*}$ via onedimensional exhaustive search, i.e., bisection search.

c) Inner level output: $\mathbf{W}^{*}, \bar{T}_{b}^{*}$, and $t_{b}^{*}$.

3) $q^{(j)}=\frac{B \log _{2}\left(1+\frac{\operatorname{Tr}\left(\mathbf{h h}^{H} \mathbf{W}^{*}\right)}{\theta f \operatorname{Tr}\left(\mathbf{g g}^{H} \mathbf{W}\right)+\sigma_{u}^{2}}\right)+\chi\left(\mathbf{W}^{*}\right)}{\xi \operatorname{Tr}\left(\mathbf{W}^{*}\right)+P_{c}}$

4) If $\left|q^{(j)}-q^{(j-1)}\right|<\rho$, then

- Algorithm Converges.

5) Else

- $\mathrm{j}=\mathrm{j}+1$, go to Step 2.

6) Outer level output: $q^{*}$.

The convergence of Algorithm 2 and the tightness of the SDP relaxed solution to problem (40) can be investigated in a similar manner to Section III-A, which is omitted here.

2) CSR: We first employ the SDP relaxation (i.e., $\mathbf{W}=$ $\mathbf{w} \mathbf{w}^{H}$ ) to modified problem (21) which is given by (41) on the top of the next page. It is easily observed that (41) is a nonconvex problem in terms of the fractional objective function (41a) and the constraint (41c), which cannot be solved directly. 


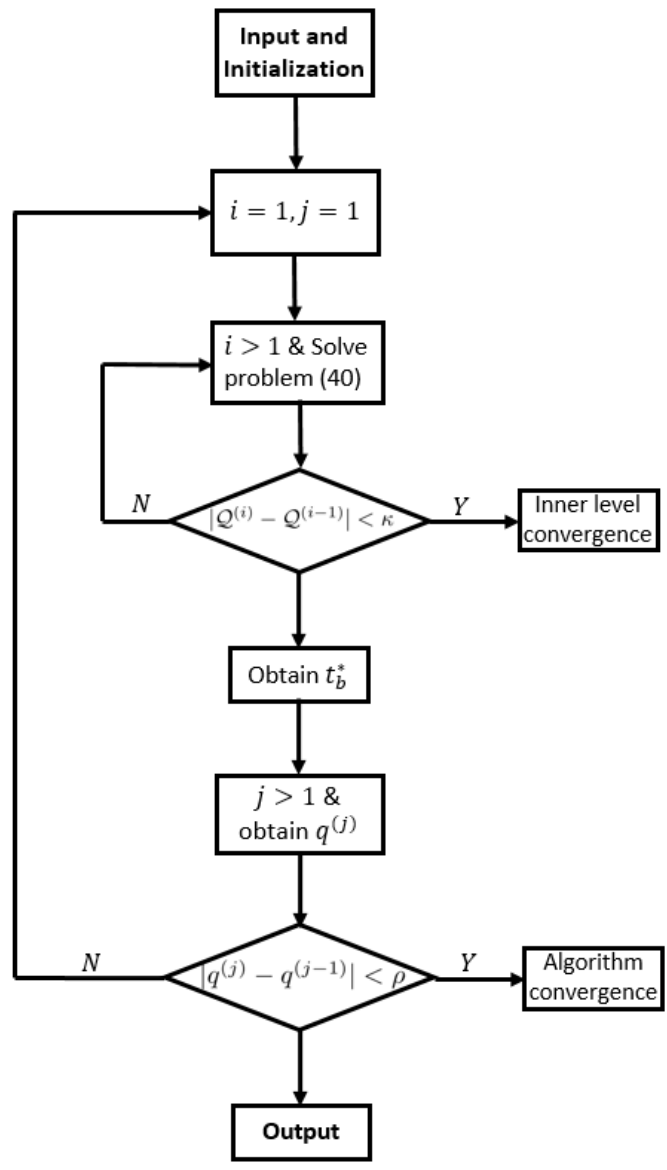

Fig. 5: Flowchart of Algorithm 2.

Similar to Section III-A2 and Section III-B1, the fractional objective function can be transformed into the subtractive form with the SCA approach to approximate the achievable rate of the backscatter link. Also, the constraint (41c) can be approximated at the $i$-th iteration via the SCA. On this basis, problem (41) can be reformulated as (42) on the top of the next page. Problem (42) is jointly non-convex and intractable. In order solve it, we employ the approach similar to Algorithm 2 in Section III-B1, which is omitted here.

\section{LOW Complexity Resource Allocation}

In Section III, we investigated the optimal resource allocation for the NSR and CSR systems via solving the transmit power minimization and energy efficiency maximization problems, respectively. The computational complexity of the solutions mainly depends on the transmit beamforming matrix W. Specifically, the complexity of the formulated problems drastically increases with the number of the transmit antennas $N_{T}$ which leads to the increased dimension of the transmit beamforming matrix $\mathbf{W}$. In order to circumvent this issue, we employ a lower complexity resource allocation scheme to solve the formulated problems. We first construct the optimal beamforming $\mathbf{w}^{*}=\mu \hat{\mathbf{h}}+\nu \hat{\mathbf{g}}$ [2], where $\hat{\mathbf{h}}=\frac{\mathbf{h}}{\|\mathbf{h}\|}$ and $\hat{\mathbf{g}}=\frac{\mathbf{g}}{\|\mathbf{g}\|}$ are the normalized channel coefficients, also $\mu$ and $\nu$ denote the complex weight factor to satisfy $|\mu|^{2}+|\nu|^{2}=1$. The optimal beamforming $\mathrm{w}^{*}$ spans these two normalized channel vectors $\hat{\mathbf{h}}$ and $\hat{\mathrm{g}}$.

In this section, we take the transmit power minimization problem for the NSR and CSR systems as an example to demonstrate this low-complexity transmit beamforming. The optimal beamforming can be equivalently modified as

$$
\tilde{\mathbf{w}}=\mathbf{A d},
$$

where $\mathbf{A}=\left[\begin{array}{ll}\hat{\mathbf{h}} & \hat{\mathbf{g}}\end{array}\right] \in \mathbb{C}^{N_{T} \times 2}$ and $\mathbf{d}=\left[\begin{array}{ll}\mu & \nu\end{array}\right]^{T} \in \mathbb{C}^{2 \times 1}$. We substitute (43) into the formulated transmit power minimization problem for the NSR and CSR systems. By employing the SDP relaxation $\tilde{\mathbf{W}}=\tilde{\mathbf{w}} \tilde{\mathbf{w}}^{H}$, we have

1) NSR: For the NSR system, the transmit power minimization problem is reformulate as

$$
\begin{aligned}
& \min _{\mathbf{D} \succeq \mathbf{0}} \operatorname{Tr}\left(\mathbf{A} \mathbf{D} \mathbf{A}^{H}\right), \text { s.t. } \operatorname{rank}(\mathbf{D})=1, \\
& \operatorname{Tr}\left(\mathbf{A}^{H} \mathbf{h} \mathbf{h}^{H} \mathbf{A D}\right)-\left(2^{\frac{R_{u}}{B}}-1\right) \theta f \operatorname{Tr}\left(\mathbf{A}^{H} \mathbf{g} \mathbf{g}^{H} \mathbf{A D}\right) \\
& \quad-\left(2^{\frac{R u}{B}}-1\right) \sigma_{u}^{2} \geq 0, \\
& \theta f \operatorname{Tr}\left(\mathbf{A}^{H} \mathbf{g g}^{H} \mathbf{A D}\right) \geq \tilde{\Upsilon}_{b}\left(\frac{S}{T}\right) \sigma_{u}^{2},
\end{aligned}
$$

where $\mathbf{D}=\mathbf{d d}^{H}, \tilde{\Upsilon}_{b}\left(\frac{S}{T}\right)$ is the root of the following equation:

$$
\begin{aligned}
& B(1-\epsilon)\left\{\frac{1}{\ln 2} \exp \left(\frac{\sigma_{u}^{2}}{\theta f \operatorname{Tr}\left(\mathbf{A}^{H} \mathbf{g g}^{H} \mathbf{A D}\right)}\right)\right. \\
& * E_{1}\left(\frac{\sigma_{u}^{2}}{\theta f \operatorname{Tr}\left(\mathbf{A}^{H} \mathbf{g g}^{H} \mathbf{A D}\right)}\right)-\beta+\frac{\sigma_{u}^{2} \beta}{2 \theta f \operatorname{Tr}\left(\mathbf{A}^{H} \mathbf{g g}^{H} \mathbf{A D}\right)} \\
& *\left[-\frac{\sigma_{u}^{2}}{\theta f \operatorname{Tr}\left(\mathbf{A}^{H} \mathbf{g g}^{H} \mathbf{A D}\right)} \exp \left(\frac{\sigma_{u}^{2}}{\theta f \operatorname{Tr}\left(\mathbf{A}^{H} \mathbf{g g}^{H} \mathbf{A D}\right)}\right)\right. \\
& \left.\left.* E_{1}\left(\frac{\sigma_{u}^{2}}{\theta f \operatorname{Tr}\left(\mathbf{A}^{H} \mathbf{g g}^{H} \mathbf{A D}\right)}\right)+1\right]\right\}=\frac{S}{T} .
\end{aligned}
$$

Problem (44) is a standard SDP without the non-convex rank-one constraint, which can be solved via interior point methods.

2) CSR: For the CSR system, the transmit power minimization problem can be approximated via the SCA, at the $i$-th iteration, as (45) on the top of the next page. By removing non-convex rank-one constraint, problem (45) can be solved via the SCA which is similar to Algorithm 1.

It is observed that the variable in both problems (44) and (45) is the matrix $\mathbf{D} \in \mathbb{C}^{2 \times 2}$ and the computational complexity is independent of the number of transmit antenna $N_{T}$ at the BS. While, the optimal resource allocation scheme in Section III has to optimize a transmit beamforming matrix $\mathbf{W} \in \mathbb{C}^{N_{T} \times N_{T}}$, and its computation complexity is dependent with $N_{T}$. Consequently, problems (44) and (45) leads to a significant reduction in computational complexity especially $N_{T}$ becomes larger. Similarly, the energy efficiency maximization problem for both NSR and CSR systems can be solved via the same beamforming structure, which is omitted here.

\section{Numerical Results}

In this section, the numerical results are presented to validate our proposed resource allocation schemes. It is assumed 


$$
\begin{aligned}
\max _{\mathbf{W} \succeq \mathbf{0}} & \frac{B \mathbb{E}_{s_{b}}\left(1+\frac{\operatorname{Tr}\left(\overline{\mathbf{h}} \overline{\mathbf{h}}^{H} \mathbf{W}\right)}{\sigma_{u}^{2}}\right)+\frac{B(1-\epsilon)}{L} \log _{2}\left[\left(1+\frac{L \theta|f|^{2} \operatorname{Tr}\left(\mathbf{g g}^{H} \mathbf{W}\right)}{\sigma_{u}^{2}}\right)-\beta \sqrt{\left.\left(1-\frac{1}{\left(1+\frac{L \theta|f|^{2} \operatorname{Tr}\left(\mathbf{g g}^{H} \mathbf{W}\right)}{\sigma_{u}^{2}}\right)^{2}}\right)\right]}\right.}{\xi \operatorname{Tr}(\mathbf{W})+P_{c}}, \\
\text { s.t. } \operatorname{Tr}(\mathbf{W}) \leq P_{\max }, & B \mathbb{E}_{s_{b}} \log _{2}\left[\left(1+\frac{\operatorname{Tr}\left(\overline{\mathbf{h}} \overline{\mathbf{h}}^{H} \mathbf{W}\right)}{\sigma_{u}^{2}}\right)\right] \geq R_{u}, \\
& \frac{B(1-\epsilon)}{L} \log _{2}\left[\left(1+\frac{L \theta|f|^{2} \operatorname{Tr}\left(\mathbf{g g}^{H} \mathbf{W}\right)}{\sigma_{u}^{2}}\right)-\beta \sqrt{\left.\left(1-\frac{1}{\left(1+\frac{L \theta|f|^{2} \operatorname{Tr}\left(\mathbf{g g}^{H} \mathbf{W}\right)}{\sigma_{u}^{2}}\right)^{2}}\right)\right]} \geq \frac{S}{T},\right.
\end{aligned}
$$

$$
\begin{aligned}
& \max _{\mathbf{W} \succeq \mathbf{0}, q} B \mathbb{E}_{s_{b}} \log _{2}\left(1+\frac{\operatorname{Tr}\left(\overline{\mathbf{h}} \overline{\mathbf{h}}^{H} \mathbf{W}\right)}{\sigma_{u}^{2}}\right)+\frac{B(1-\epsilon)}{L}\left\{\log _{2}\left(1+\frac{L \theta|f|^{2} \operatorname{Tr}\left(\mathbf{g g}^{H} \mathbf{W}\right)}{\sigma_{u}^{2}}\right)\right. \\
& -\beta\left[\sqrt{1-\frac{1}{\left(1+\frac{L \theta|f|^{2} \operatorname{Tr}\left(\mathbf{g g}^{H} \mathbf{W}^{(i-1)}\right)}{\sigma_{u}^{2}}\right)^{2}}}+\frac{1}{\left(1+\frac{L \theta|f|^{2} \operatorname{Tr}\left(\mathbf{g g}^{H} \mathbf{W}^{(i-1)}\right)}{\sigma_{u}^{2}}\right)^{2}} \frac{1}{\sqrt{\left(1+\frac{L \theta|f|^{2} \operatorname{Tr}\left(\mathbf{g g}^{H} \mathbf{W}^{(i-1)}\right)}{\sigma_{u}^{2}}\right)^{2}-1}}\right. \\
& \left.\left.\quad *\left[1+\frac{L \theta|f|^{2} \operatorname{Tr}\left(\mathbf{g g}^{H} \mathbf{W}\right)}{\sigma_{u}^{2}}-\left(1+\frac{L \theta|f|^{2} \operatorname{Tr}\left(\mathbf{g g}^{H} \mathbf{W}^{(i-1)}\right)}{\sigma_{u}^{2}}\right)\right]\right]\right\}-q\left(\xi \operatorname{Tr}(\mathbf{W})+P_{c}\right), \\
& \text { s.t. } \operatorname{Tr}(\mathbf{W}) \leq P_{\max }, \\
& \quad B \mathbb{E}_{s_{b}} \log _{2}\left[\left(1+\frac{\operatorname{Tr}\left(\overline{\mathbf{h}} \overline{\mathbf{h}}^{H} \mathbf{W}\right)}{\sigma_{u}^{2}}\right)\right] \geq R_{u},(30),
\end{aligned}
$$

$$
\begin{aligned}
& \min _{\mathbf{D} \succeq \mathbf{0}} \operatorname{Tr}\left(\mathbf{A D} \mathbf{A}^{H}\right), \text { s.t. } \operatorname{rank}(\mathbf{D})=1, \\
& B \mathbb{E}_{s_{b}}\left[\log _{2}\left(1+\frac{\operatorname{Tr}\left(\mathbf{A}^{H} \overline{\mathbf{h}} \overline{\mathbf{h}}^{H} \mathbf{A D}\right)}{\sigma_{u}^{2}}\right)\right] \geq R_{u}, \\
& \frac{B(1-\epsilon)}{L}\left\{\log _{2}\left(1+\frac{L \theta|f|^{2} \operatorname{Tr}\left(\mathbf{A}^{H} \mathbf{g g}{ }^{H} \mathbf{A D}\right)}{\sigma_{u}^{2}}\right)-\beta\left[\sqrt{1-\frac{1}{\left(1+\frac{L \theta|f|^{2} \operatorname{Tr}\left(\mathbf{A}^{H} \mathbf{g g}^{H} \mathbf{A D}^{(i-1)}\right)}{\sigma_{u}^{2}}\right)^{2}}}\right.\right. \\
& +\frac{1}{\left(1+\frac{L \theta|f|^{2} \operatorname{Tr}\left(\mathbf{A}^{H} \mathbf{g g}^{H} \mathbf{A} \mathbf{D}^{(i-1)}\right)}{\sigma_{u}^{2}}\right)^{2}} \frac{1}{\sqrt{\left(1+\frac{L \theta|f|^{2} \operatorname{Tr}\left(\mathbf{A}^{H} \mathbf{g g}^{H} \mathbf{A} \mathbf{D}^{(i-1)}\right)}{\sigma_{u}^{2}}\right)^{2}-1}}\left[1+\frac{L \theta|f|^{2} \operatorname{Tr}\left(\mathbf{A}^{H} \mathbf{g g}^{H} \mathbf{A D}\right)}{\sigma_{u}^{2}}\right. \\
& \left.\left.\left.-\left(1+\frac{L \theta|f|^{2} \operatorname{Tr}\left(\mathbf{A}^{H} \mathbf{g g}^{H} \mathbf{A} \mathbf{D}^{(i-1)}\right)}{\sigma_{u}^{2}}\right)\right]\right]\right\} \geq \frac{S}{T} .
\end{aligned}
$$

that the BS is equipped with four transmit antennas $N_{T}=4$, while the $\mathrm{BD}$ and the receiver consist of single-antenna. All channel coefficients consist of small-scale and large-scale parts. The small-scale channel coefficients are generated as the identical and circularly complex Gaussian random variable with zero mean and unit variance. The large-scale path loss is dependent on the distance between any two nodes, which is given by $P L=\bar{d}^{-\alpha}$, where $\bar{d}$ represents the communication link distance from BS to BD (i.e., $d_{B S 2 B D}$ ), from BS to receiver (i.e., $d$ ), from $\mathrm{BD}$ to receiver (i.e., $d_{B D 2 R}$ ), respectively, and $\alpha$ denotes the path loss exponent. As illustrated in Fig.
6 , it is a 2-D coordinate to describe that the coordinates of the BS, the receiver and the BD are located at $(0,0),(d, 0)$, and $\left(d_{1},-d_{p}\right)$ in meter $(\mathrm{m})$. The distance between the BS and the receiver is set to $d=30 \mathrm{~m}$, and the distance of the vertical line is assumed $d_{p}=5 \mathrm{~m}$. The horizontal distance between the $\mathrm{BS}$ and $\mathrm{BD}$ is denoted by $d_{1}$, and the horizontal distance between the BD and the receiver is $d_{2}=d-d_{1}$, which affect the power consumption and the energy efficiency performances. In the scenario shown in Fig. 6, the distances between $\mathrm{BS}$ and $\mathrm{BD}$ is set to be $d_{B S 2 B D}=\sqrt{d_{1}^{2}+\left(-d_{p}\right)^{2}}$ and the distance between $\mathrm{BD}$ and receiver is set to be 


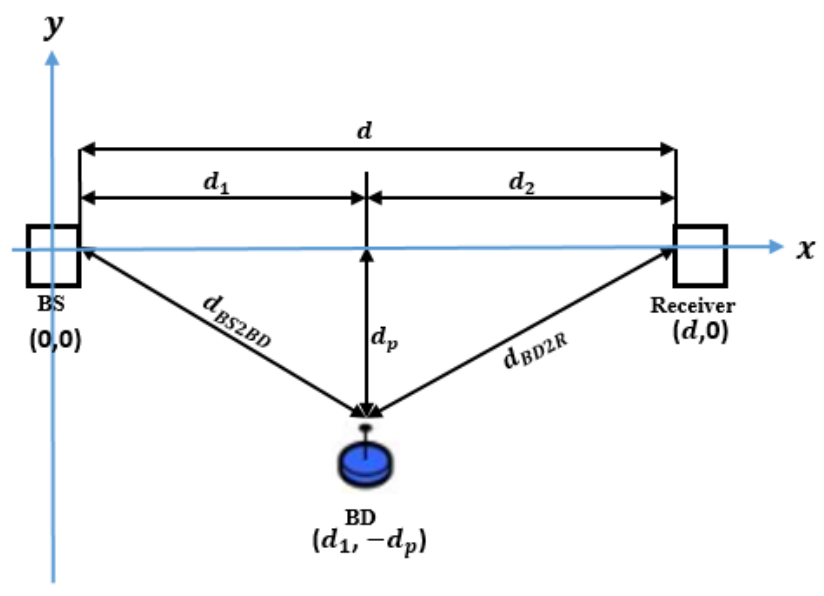

Fig. 6: System deployment.

$d_{B D 2 R}=\sqrt{\left(d-d_{1}\right)^{2}+\left(-d_{p}\right)^{2}}$. The path loss exponents of $\mathrm{BS} 2 \mathrm{R}$ link, BS2BD link, as well as BD2R link are set to $\alpha_{B S 2 R}=3.5$, and $\alpha_{B S 2 B D}=\alpha_{B D 2 R}=2.5$, respectively. In addition, we assume that the bandwidth $B$ is set to be $10 \mathrm{MHz}$, the noise power spectral density is $-120 \mathrm{dBm} / \mathrm{Hz}$, the circuit power $P_{c}=0.1 \mathrm{~W}$, the reflection coefficient is $\theta=0.6$, packet size is $S=500$ byte, $T=10^{-3}$ s, code block-length $m=200$, block error probability $\epsilon=10^{-3}$ unless specified.

\section{A. Transmit Power Minimization}

We first evaluate the transmit power minimization problem for the NSR and CSR schemes. Fig. 7 shows that the transmit power versus the achievable rate of the direct link. It is observed from this figure that for the direct link in both NSR and CSR schemes, the power consumption increases with the achievable rate. Also, the low complexity resource allocation scheme achieves the same performance with the proposed scheme. In addition, we compare the proposed NSR and CSR schemes with the scheme without BD. Fig. 7 shows that the proposed CSR scheme has a better performance than that without $\mathrm{BD}$ in terms of power consumption, while the scheme without BD has slight power saving compared to the NSR scheme. This is due to the fact that the backscatter link in the NSR scheme introduces the interference to the direct link, leading to an increased power consumption. Whereas, in the CSR scheme, both backscatter and direct links may be beneficial from each other without interference due to additional multipath diversity, leading to more power efficient transmissions. We also compare the proposed scheme with the bandwidth allocation scheme [32], under the same configuration here. Apparently, the bandwidth allocation scheme is not as good as both proposed schemes. This is owing to the fact that the direct and backscatter links are allocated smaller bandwidths thus affecting the power consumption.

In Fig. 8, we evaluate the transmit power versus the horizon distance between the $\mathrm{BS}$ and the $\mathrm{BD} d_{1}$. From this figure, one can observe that the transmission power of the NSR scheme slightly changes with $d_{1}$. Specifically, it initially has an inconspicuous decrease and then increases. This is due to the

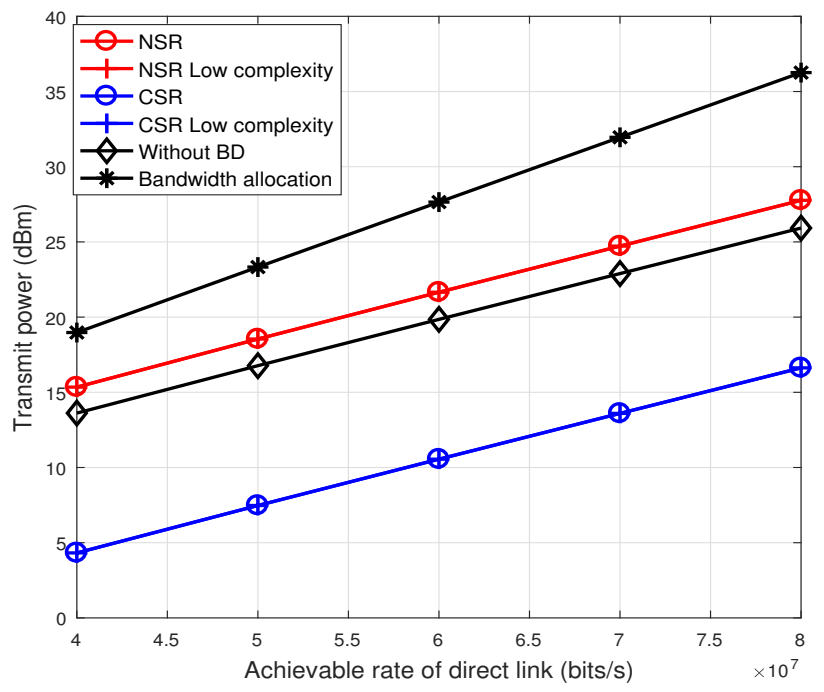

Fig. 7: Transmit power versus achievable rate of direct link.

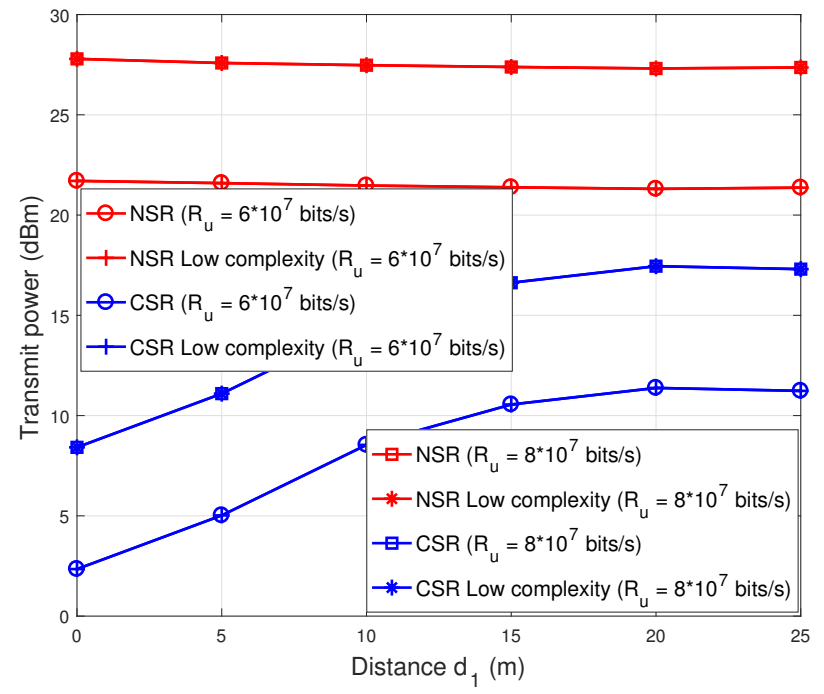

Fig. 8: Transmit power versus horizon distance between BS and BD $d_{1}$

fact that larger distance $d_{1}$ will lead to smaller interference of the backscatter link such that the NSR scheme has less power consumption. Whereas, as $d_{1}$ increases, the BD moves nearer to the receiver which results in a larger interference of the backscatter link to affect the direct link. This will bring more power consumption in the NSR scheme. Whereas, the CSR scheme experiences an obvious change with $d_{1}$. It has an increasing trend and then decline when $d_{1} \geq 20 \mathrm{~m}$. This is owing to the fact that the BD is placed farther away to the BS such that the CSR scheme consumes more power, whereas the $\mathrm{BD}$ moves closer to the receiver so as to play a cooperative role to improve the power efficiency performance.

Fig. 9 shows that the transmit power versus delay requirement $T$. It is clearly observed that both NSR and CSR schemes has a slightly decreasing trend and then keeps stable 


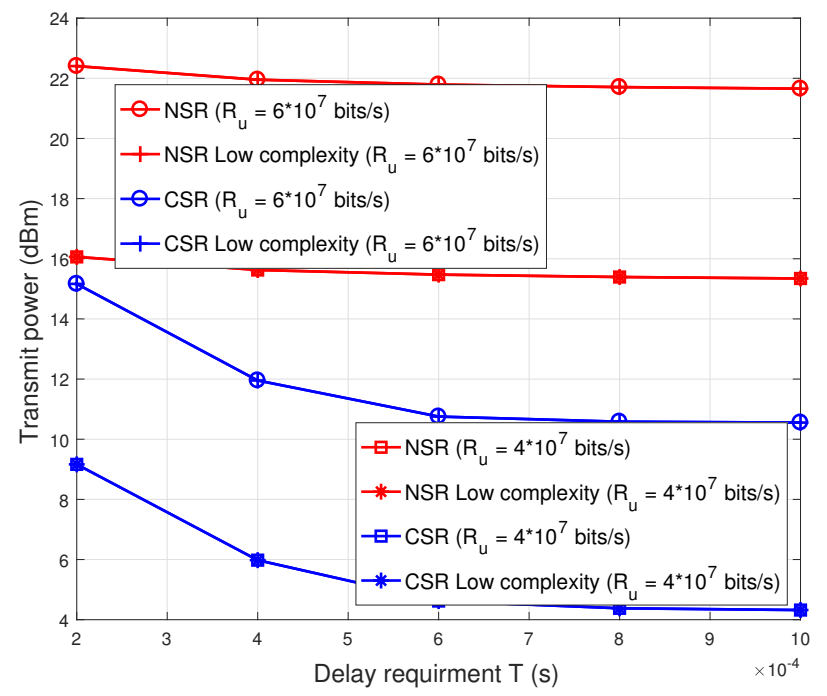

Fig. 9: Transmit power versus delay requirement $T$.

as $T$ increases. The reason leading to this trend is that higher delay requirement indicates a lower achievable rate of backscatter link, which improves the transmit power efficiency performance.

Fig. 10 shows that the power consumption versus the number of transmit antenna at the BS $N_{T}$. From this figure, it is observed that the power consumption has a decreasing trend with $N_{T}$ for each scheme. Also, it can also be observed from this figure that the proposed CSR scheme outperforms the one without BD in terms of power consumption, while the scheme without BD has slight power saving compared to the NSR scheme. Fig. 11 shows the power consumption comparison with the horizontal distance $d_{1}$ and the achievable rate of the direct link for the NSR and CSR systems. It is seen from this figure that the power consumption has an increasing trend with the achievable rate of the direct link in both NSR and CSR schemes, where the CSR scheme outperforms the NSR scheme. From this figure, it can also be observed that the power consumption in the NSR scheme has an initially inconspicuous decrease and then slightly increases with $d_{1}$. Whereas, the CSR scheme experiences an obviously increasing trend and then a slightly decline with $d_{1}$.

\section{B. Energy Efficiency Maximization}

Then, we evaluate the maximum energy efficiency performance for the NSR and CSR schemes. ${ }^{3}$ Fig. 12 shows that the energy efficiency versus transmit power. From this figure, one can observe that the energy efficiency in both NSR and CSR schemes has an increasing trend with the target transmit power and then keep constant when transmit power is greater than $22 \mathrm{dBm}$. It indicates that it is not suitable to spend full power from energy efficiency perspective. Also, we compare the proposed scheme with the scheme in [2], i.e., spectral efficiency scheme, under the same configurations. It

\footnotetext{
${ }^{3}$ We denote the energy efficiency and spectral efficiency by "EE" and 'SE" in the legend of the simulation results.
}

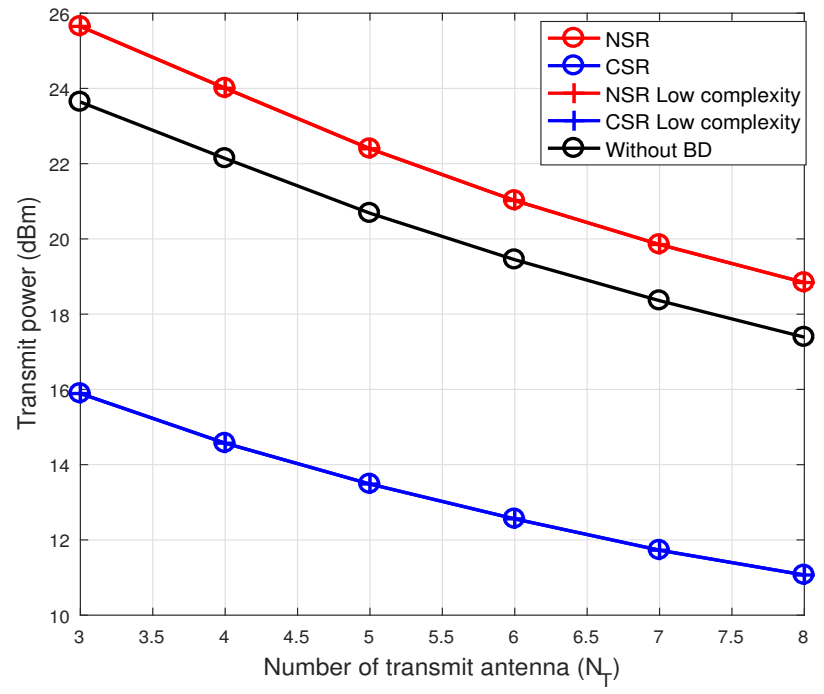

Fig. 10: Transmit power versus number of transmit antenna $N_{T}$.

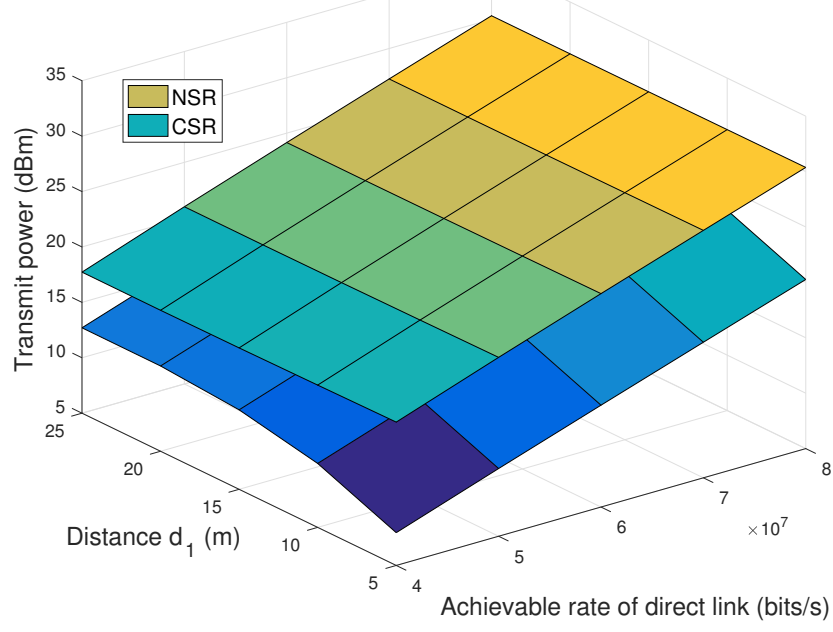

Fig. 11: Power consumption comparison of NSR and CSR systems.

is observed that the spectral efficiency scheme has a same trend with the energy efficiency scheme as transmit power increases. After approximately $22 \mathrm{dBm}$, the energy efficiency scheme has an obvious advantage over the spectral efficiency scheme.

Fig. 13 shows that the energy efficiency performance versus the horizon distance between the $\mathrm{BS}$ and the $\mathrm{BD} d_{1}$. From this figure, one can observe that the NSR scheme slightly changes with $d_{1}$, specifically, first it has an inconspicuous increase and then slightly decreases. This is due to the fact that larger distance $d_{1}$ will lead to smaller interference of the backscatter, thus slightly improved energy efficiency. Whereas, as $d_{1}$ increases, the BD moves nearer to the receiver which results in a larger interference of the backscatter link to affect the direct link, leading to a slightly decreased energy efficiency. However, the CSR scheme experiences a conspicuous change 


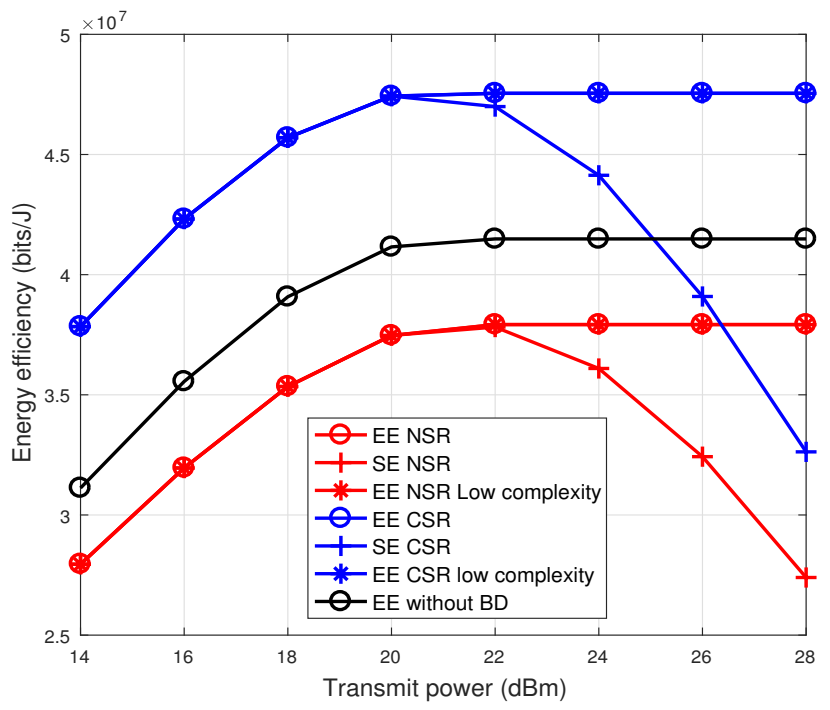

Fig. 12: Energy efficiency versus transmit power.

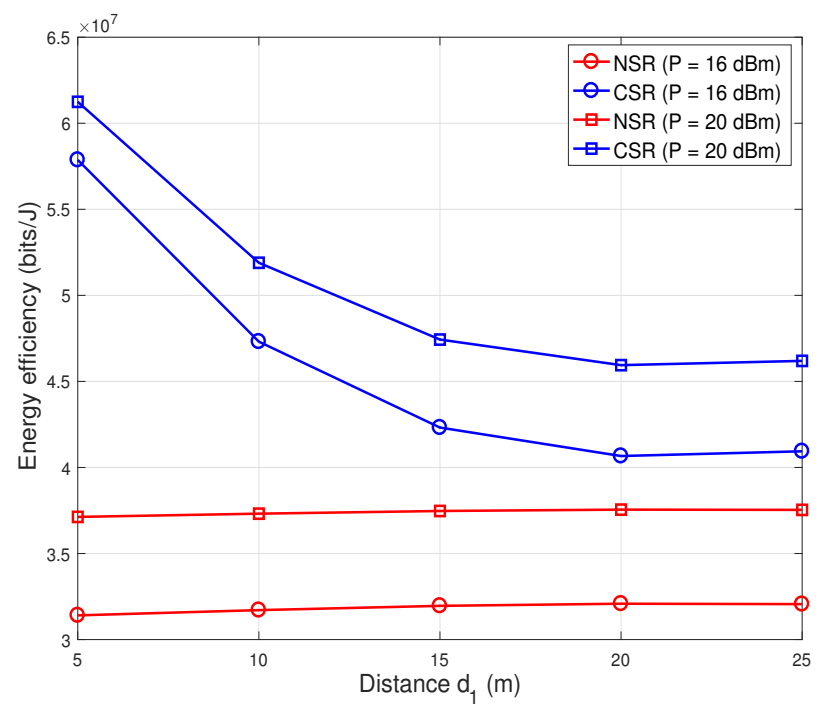

Fig. 13: Energy efficiency versus horizon distance between BS and $\mathrm{BD} d_{1}$.

with $d_{1}$. It has an decreasing trend and then increases when $d_{1} \geq 20 \mathrm{~m}$. This is owing to the fact that the BD is placed farther away to the BS leading to a smaller energy efficiency, whereas the BD moves nearer to the receiver so as to play a cooperative role to improve the energy efficiency performance.

In Fig. 14, we evaluate the energy efficiency performance versus the number of transmit antenna at the BS $N_{T}$, in which we can observe that the energy efficiency has an increasing trend with $N_{T}$ for every scheme, and reaches a point of diminishing returns in the larger $N_{T}$ regime. In Fig. 15, we evaluate the energy efficiency performance comparison between the NSR and CSR schemes. From this figure, similar arguments from Fig. 12 and Fig. 13 can also be observed here that the energy efficiency in both NSR and CSR schemes has

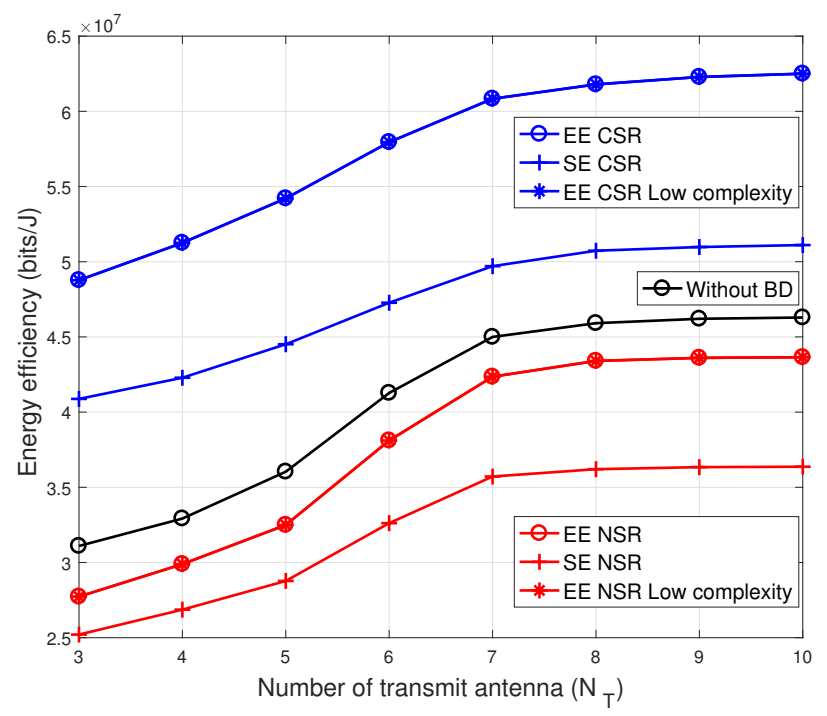

Fig. 14: Energy efficiency versus number of transmit antenna $N_{T}$.

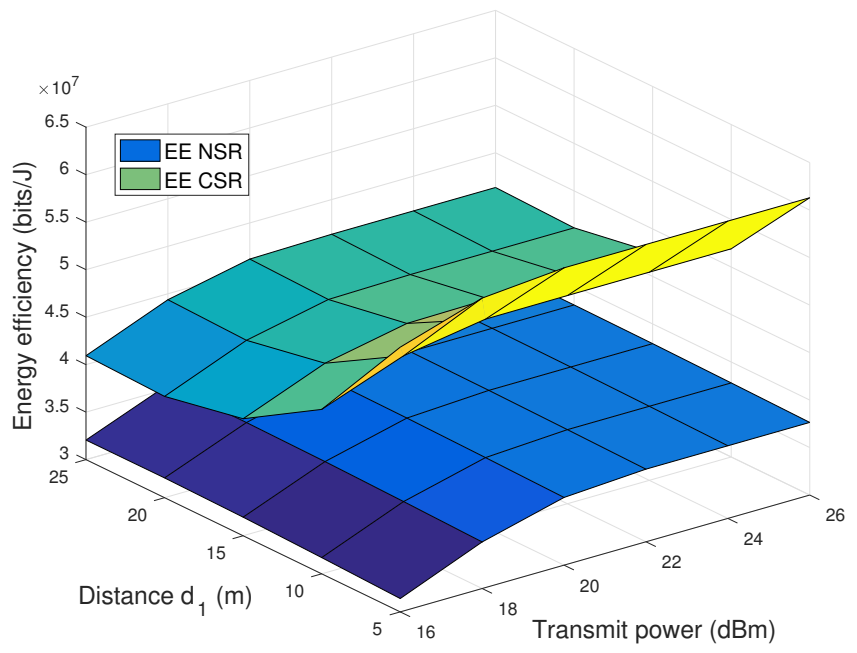

Fig. 15: Energy efficiency comparison of NSR and CSR systems.

an increasing trend with the transmit power and then remains constant in the large power regime. Also, the NSR scheme initially has an inconspicuous increase and then slightly decreases with $d_{1}$, however, the CSR scheme experiences a conspicuous change with $d_{1}$, which has an decreasing trend and then slightly decline. It is also seen that the CSR scheme has a better performance than the NSR scheme in terms of the energy efficiency performance.

Finally, we evaluate the convergence of Algorithm 2. From Fig. 16, it is clearly observed that the energy efficiency in both NSR and CSR schemes increases first and converges to a stable value, which validates the convergence of Algorithm 2 . 


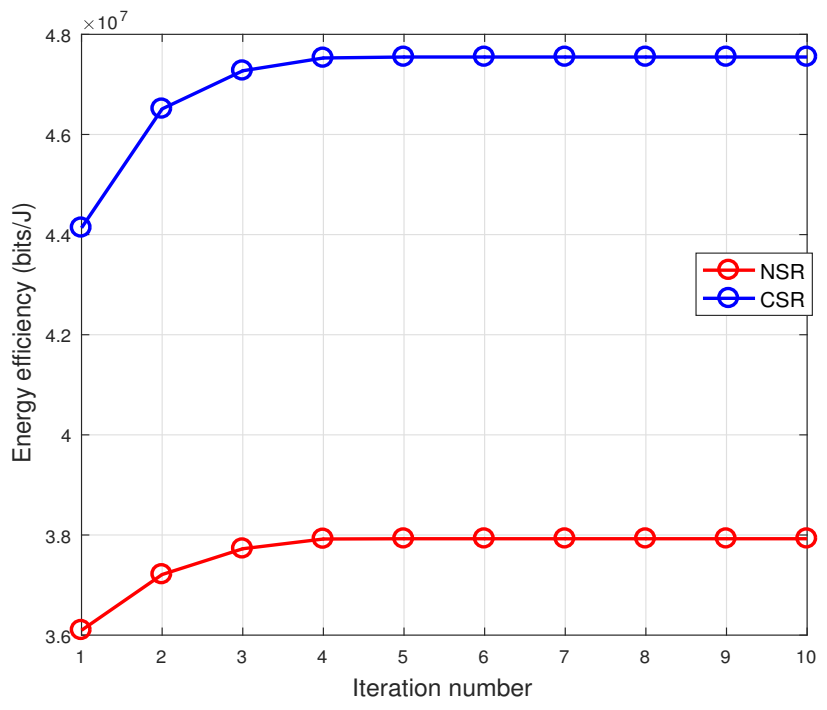

Fig. 16: Convergence performance of energy efficiency.

\section{CONCLUSION}

This paper studied a downlink MISO SR system, where the BS coordinates the direct and backscatter links, and the receiver decodes the information from the $\mathrm{BS}$ and the $\mathrm{BD}$. The backscattered signal packets are designed to have finite block-length to accurately measured backscatter link. The backscatter transmission rate was measured in the finite blocklength channel codes. We investigated the NSR and CSR systems from different types of the backscatter symbol period and transmission rate, and derived their average achievable rate of the direct and backscatter links, respectively. The energy efficient power allocation problems, i.e., transmit power minimization and energy efficiency maximization, have been formulated. The SDP relaxation and SCA were considered to design the transmit beamforming vector. Moreover, a lowcomplexity transmit beamforming structure was constructed to reduce the computation complexity of the SDP relaxed solution. Finally, the simulation results were presented to demonstrate the effectiveness of the proposed schemes, and the CSR scheme was shown to outperform the NSR scheme and the case without BD in terms of power consumption and energy efficiency.

In this paper, we consider the resource allocation for the SR system with the finite block-length backscatter link. For future works, we can investigate a more challenging and practical scenario that both direct and backscatter links are considered to be finite block-length. This would change the dynamic of the optimization problems and may require different designs/solutions. In addition, the design based on the finite alphabet inputs can circumvent the performance loss due to the assumption of the Gaussian inputs in the SR system.

\section{REFERENCES}

[1] L. Zhang, Y. Liang, and M. Xiao, "Spectrum sharing for internet of things: A survey," IEEE Wireless Commun., vol. 26, no. 3, pp. 132 139, Jun. 2019.
[2] R. Long, Y. Liang, H. Guo, G. Yang, and R. Zhang, "Symbiotic radio: A new communication paradigm for passive internet-of-things," to appear in IEEE Internet Things J., pp. 1-14, 2019.

[3] V. Liu, A. Parks, V. Talla, S. Gollakota, D. Wetherall, and J. R. Smith, "Ambient backscatter: Wireless communication out of thin air," in Proc. ACM SIGCOMM, Hong Kong, China, pp. 39-50, 2013.

[4] C. Boyer and S. Roy, "Backscatter communication and RFID: Coding, energy, and MIMO analysis," IEEE Transactions on Communications, vol. 62, no. 3, pp. 770-785, Mar. 2014.

[5] G. Wang, F. Gao, R. Fan, and C. Tellambura, "Ambient backscatter communication systems: Detection and performance analysis," IEEE Trans. Commun., vol. 64, no. 11, pp. 4836-4846, Nov. 2016.

[6] R. Long, G. Yang, Y. Pei, and R. Zhang, "Transmit beamforming for cooperative ambient backscatter communication systems," in Proc. IEEE GLOBECOM, Singapore, pp. 1-6, Dec 2017.

[7] J. Kimionis, A. Bletsas, and J. N. Sahalos, "Increased range bistatic scatter radio," IEEE Trans. Commun., vol. 62, no. 3, pp. 1091-1104, Mar. 2014.

[8] J. F. Ensworth and M. S. Reynolds, "Every smart phone is a backscatter reader: Modulated backscatter compatibility with bluetooth 4.0 low energy (ble) devices," in Proc. IEEE Intl Conf. RF, San Diego, CA, USA, pp. 78-85, Apr. 2015.

[9] V. Talla, M. Hessar, B. Kellogg, A. Najafi, J. R. Smith, and S. Gollakota, "LoRa backscatter: Enabling the vision of ubiquitous connectivity," Proc. ACM Interact. Mob. Wearable Ubiquitous Techn, vol. 1, no. 3, pp. 105:1-105:24, Sept. 2017.

[10] H. Guo, Q. Zhang, S. Xiao, and Y. Liang, "Exploiting multiple antennas for cognitive ambient backscatter communication," IEEE Internet Things J., vol. 6, no. 1, pp. 765-775, Feb. 2019.

[11] G. Yang, Y. Liang, R. Zhang, and Y. Pei, "Modulation in the air: Backscatter communication over ambient OFDM carrier," IEEE Trans. Commun., vol. 66, no. 3, pp. 1219-1233, Mar. 2018.

[12] G. Yang, Q. Zhang, and Y. Liang, "Cooperative ambient backscatter communications for green internet-of-things," IEEE Internet Things J., vol. 5, no. 2, pp. 1116-1130, Apr. 2018

[13] X. Kang, Y. Liang, and J. Yang, "Riding on the primary: A new spectrum sharing paradigm for wireless-powered IoT devices," IEEE Trans. Wireless Commun., vol. 17, no. 9, pp. 6335-6347, Sept. 2018.

[14] W. Liu, Y. Liang, Y. Li, and B. Vucetic, "Backscatter multiplicative multiple-access systems: Fundamental limits and practical design," IEEE Transactions on Wireless Communications, vol. 17, no. 9, pp. 57135728, Sept. 2018

[15] H. Guo, Y. Liang, R. Long, S. Xiao, and Q. Zhang, "Resource allocation for symbiotic radio system with fading channels," IEEE Access, vol. 7 , pp. 34333-34347, Apr. 2019.

[16] H. Guo, Y. Liang, R. Long, and Q. Zhang, "Cooperative ambient backscatter system: A symbiotic radio paradigm for passive IoT," IEEE Wireless Commun. Lett., vol. 8, no. 4, pp. 1191-1194, Aug. 2019.

[17] R. Long, H. Guo, L. Zhang, and Y. Liang, "Full-duplex backscatter communications in symbiotic radio systems," IEEE Access, vol. 7, pp. 21597-21608, Feb. 2019.

[18] R. Long, H. Guo, and Y. Liang, "Symbiotic radio with full-duplex backscatter devices," in IEEE ICC, Shanghai, China, pp. 1-6, May 2019.

[19] Q. Zhang, L. Zhang, Y. Liang, and P. Kam, "Backscatter-NOMA: A symbiotic system of cellular and internet-of-things networks," IEEE Access, vol. 7, pp. 20000-20013, Feb. 2019.

[20] Q. Zhang, Y. Liang, and H. V. Poor, "Intelligent user association for symbiotic radio networks using deep reinforcement learning," arXiv, vol. https://arxiv.org/abs/1905.04041, 2019.

[21] Y. Polyanskiy, H. V. Poor, and S. Verdu, "Channel coding rate in the finite blocklength regime," IEEE Trans. Inf. Theory, vol. 56, no. 5, pp. 2307-2359, May 2010.

[22] G. Durisi, T. Koch, and P. Popovski, "Toward massive, ultra-reliable, and low-latency wireless communication with short packets," Proc. IEEE, vol. 104, no. 9, pp. 1711-1726, Sept. 2016.

[23] Y. Li, M. C. Gursoy, and S. Velipasalar, "Throughput of two-hop wireless channels with queueing constraints and finite blocklength codes," in Proc. ISIT, Barcelona, Spain, pp. 2599-2603, Jul. 2016.

[24] Y. Hu, M. C. Gursoy, and A. Schmeink, "Relaying-enabled ultra-reliable low-latency communications in 5G," IEEE Netw., vol. 32, no. 2, pp. 62 68, Mar. 2018.

[25] X. Sun, S. Yan, N. Yang, Z. Ding, C. Shen, and Z. Zhong, "Shortpacket downlink transmission with non-orthogonal multiple access," IEEE Trans. Wireless Commun., vol. 17, no. 7, pp. 4550-4564, Jul. 2018.

[26] Z. Chu, W. Yu, P. Xiao, F. Zhou, N. Al-Dhahir, A. u. Quddus, and R. Tafazolli, "Opportunistic spectrum sharing for D2D-based URLLC," IEEE Trans. Veh. Technol., vol. 68, no. 9, pp. 8995-9006, Sept. 2019. 
[27] D. W. K. Ng, E. S. Lo, and R. Schober, "Energy-efficient resource allocation in OFDMA systems with large numbers of base station antennas," IEEE Trans. Wireless Commun., vol. 11, no. 9, pp. 32923304, Sept. 2012.

[28] S. Boyd and L. Vandenberghe, Convex Optimization. Cambridge, UK: Cambridge University Press, 2004.

[29] J. Tang, B. Shim, and T. Q. S. Quek, "Service multiplexing and revenue maximization in sliced C-RAN incorporated with URLLC and multicast eMBB," IEEE J. Sel. Areas Commun., vol. 37, pp. 881-895, April Apr. 2019

[30] M. Razaviyayn, M. Hong, and Z. Luo, "A unified convergence analysis of block successive minimization methods for nonsmooth optimization," SIAM J. Optimiz., vol. 23, no. 2, pp. 1126-1153, 2013.

[31] A. Charnes and W. W. Cooper, "Programming with linear fractional functionals," Naval Res. Logist. Quarter, vol. 9, pp. 181-186, 1962.

[32] J. Tang, B. Shim, T. Chang, and T. Q. S. Quek, "Incorporating URLLC and multicast eMBB in sliced cloud radio access network," in Proc. IEEE ICC, Shanghai, China, pp. 1-7, May 2019. 